\title{
Robust Beamforming for Secure Communication in Systems with Wireless Information and Power Transfer
}

\author{
Derrick Wing Kwan Ng, Member, IEEE, Ernest S. Lo, Member, IEEE, \\ and Robert Schober, Fellow, IEEE
}

\begin{abstract}
This paper considers a multiuser multiple-input single-output (MISO) downlink system with simultaneous wireless information and power transfer. In particular, we focus on secure communication in the presence of passive eavesdroppers and potential eavesdroppers (idle legitimate receivers). We study the design of a resource allocation algorithm minimizing the total transmit power for the case when the legitimate receivers are able to harvest energy from radio frequency signals. Our design advocates the dual use of both artificial noise and energy signals in providing secure communication and facilitating efficient wireless energy transfer. The algorithm design is formulated as a non-convex optimization problem. The problem formulation takes into account artificial noise and energy signal generation for protecting the transmitted information against both considered types of eavesdroppers when imperfect channel state information (CSI) of the potential eavesdroppers and no CSI of the passive eavesdroppers are available at the transmitter. Besides, the problem formulation also takes into account different quality of service $(\mathrm{QoS})$ requirements: a minimum required signal-tointerference-plus-noise ratio (SINR) at the desired receiver; maximum tolerable SINRs at the potential eavesdroppers; a minimum required outage probability at the passive eavesdroppers; and minimum required heterogeneous amounts of power transferred to the idle legitimate receivers. In light of the intractability of the problem, we reformulate the considered problem by replacing a non-convex probabilistic constraint with a convex deterministic constraint. Then, a semi-definite programming (SDP) relaxation approach is adopted to obtain the optimal solution for the reformulated problem. Furthermore, we propose a suboptimal resource allocation scheme with low computational complexity for providing communication secrecy and facilitating efficient energy transfer. Simulation results demonstrate the close-tooptimal performance of the proposed schemes and significant transmit power savings by optimization of the artificial noise and energy signal generation.
\end{abstract}

Index Terms-Physical (PHY) layer security, passive eavesdropper, wireless information and power transfer, artificial noise, robust beamforming.

\section{INTRODUCTION}

Manuscript received November 7, 2013; revised February 11, 2014; accepted March 26, 2014. The review of this paper was coordinated by Prof. C. Yang. This paper has been presented in part at IEEE Globecom 2013 [1] and IEEE PIMRC 2013 [2], respectively. Derrick Wing Kwan $\mathrm{Ng}$ and Robert Schober are with the Institute for Digital Communications (IDC), Friedrich-Alexander-University Erlangen-Nürnberg (FAU), Germany (email:\{kwan, schober\}@lnt.de). Ernest S. Lo is with the Centre Tecnològic de Telecomunicacions de Catalunya - Hong Kong (CTTC-HK) (email: ernest.lo@cttc.hk). This work was supported in part by the AvH Professorship Program of the Alexander von Humboldt Foundation.
$\mathbf{T}$ HE exponential growth in the demand for high data rates in wireless communication networks has led to a tremendous need for energy. The rapidly escalated energy consumption not only increases the operating cost of communication systems, but also raises serious environmental concerns. As a result, green radio communications has received much attention in both academia and industry [1] -9$]$. In particular, multiple antennas and multicarrier technologies have been proposed in the literature for facilitating energy savings in wireless communication systems. Unfortunately, the computational burden and power consumption incurred by the signal processing required for these technologies may be too high for small portable mobile units. As an alternative, multiuser multiple-input multiple-output (MIMO) has been proposed where a transmitter equipped with multiple antennas services multiple single-antenna users. However, mobile communication devices are often powered by capacity limited batteries. Hence, the lifetime of mobile devices remains the bottleneck in the development of ubiquitous wireless communication services.

The integration of energy harvesting with communication devices is considered a promising alternative in providing self-sustainability to energy limited communication systems [1]-[8]. Hydroelectric, piezoelectric, solar, and wind are the major conventional energy sources for energy harvesting. Nevertheless, the availability of these natural energy sources is usually limited by location or climate and may be problematic in indoor environments. On the other hand, ambient background electromagnetic radiation in radio frequency (RF) is also a viable source of energy for energy scavenging. More importantly, wireless energy harvesting technology facilitates the possibility of simultaneous wireless information and power transfer. Yet, this new technology introduces a paradigm shift in system and resource allocation algorithm design due to the imposed new challenges. In [3], the fundamental tradeoff between harvested energy and data rate was studied. In [4], a power allocation algorithm was proposed for near-field communication systems. However, the results from [3] and [4] were obtained by assuming that the receiver is able to recycle energy and decode information from the same received signal which is not possible yet in practice. Thus, power splitting hybrid receivers and separated receivers were proposed in [5] and [6], respectively, as a compromise solution. In particular, the power splitting receiver splits the received power into two power streams in order to facilitate simultaneous energy 
harvesting and information decoding at the receiver. In [7], the authors proposed different power allocation algorithms and showed that introducing power splitting receivers can improve the energy efficiency of a communication system. Transmitting an energy signal along with the information signal was proposed in [8] for expediting energy harvesting at the receivers. In practice, the transmitter can increase the energy of the information carrying signal to facilitate energy harvesting at the receivers. However, this may also increase the susceptibility to eavesdropping due to a higher potential for information leakage. Therefore, a new quality of service (QoS) concern on communication security arises in systems with simultaneous information and power transfer.

In fact, security is a fundamental problem in wireless communication systems due to the broadcast nature of the wireless medium. Traditionally, communication security relies on cryptographic encryption employed at the application layer. However, the commonly used encryption algorithms are based on the assumption of limited computational capability at the eavesdroppers [10], [11]. Besides, these algorithms assume a perfect secret key management and distribution which may not be possible in wireless networks. Hence, recently a large amount of work has been devoted to information-theoretic physical (PHY) layer security [12]-[16], as an alternative or complement to cryptographic encryption. The principle of PHY layer security is to exploit the physical characteristics of the wireless fading channel for providing perfect secrecy of communication. In his pioneering work on PHY layer security [12], Wyner showed that in a wire-tap channel, a source and a destination can exchange perfectly secure information at a strictly positive data rate whenever the source-destination channel enjoys better conditions compared to the sourceeavesdropper channel. Hence, exploiting multiple antennas has been proposed in the literature to ensure secure communication. In particular, by utilizing the extra degrees of freedom offered by multiple antennas, artificial noise (/interference) is injected into the communication channel to impair the received signals at the eavesdroppers. In [13] and [14], power allocation algorithms were proposed for maximizing the ergodic secrecy capacity and outage secrecy capacity via artificial noise generation, respectively. In [15], joint optimization of transmit beamforming and artificial noise was performed to ensure secure communication in a system with multiple single antenna eavesdroppers. In [16], outage-based optimization was proposed to maximize the system secrecy capacity. However, the energy sources of the receivers in [13][16] were assumed to be perpetual which may not be possible for energy constrained systems. Furthermore, artificial noise generation may consume a significant portion of the transmit power to guarantee secure communication [13]-[16]. On the other hand, the transmitted artificial noise can be harvested by the receivers and the recycled energy can be used to extend the lifetime of power-constrained portable devices. Yet, the proposed algorithms in [13]-[16] do not exploit the dual use of artificial noise for securing the communication and energy harvesting.

The notion of secure communication in energy harvesting systems has recently been pursued in different contexts. In
[1], different power allocation algorithms with artificial noise generation were designed for power splitting receivers to realize efficient concurrent power transfer and secure communication. In [2], a multi-objective approach was used to jointly maximize the energy harvesting efficiency and minimize the total transmit power while ensuring communication secrecy. Exploiting an energy signal to provide secure communication in systems with separated information and energy harvesting receivers was studied in [17], [18]. Yet, the assumption of having perfect channel state information (CSI) of the energy harvesting receivers in [1], [2], [17], and [18] may be overly optimistic. In fact, the energy harvesting receivers are not continuously interacting with the transmitter and the corresponding CSI at the transmitter may be outdated even if the channel is only slowly time varying. Besides, the energy receivers can be malicious and take advantage of the system by reporting false CSI (in frequency division duplex (FDD) systems). In [19], robust beamforming for the case of imperfect CSI was studied for energy harvesting communication systems with one energy harvesting receiver and one information receiver without considering communication security. However, it is unclear if the solution in [19] can be extended to multiple energy harvesting receivers while ensuring secure communication. Furthermore, different types of eavesdroppers may exist in the system. For instance, passive eavesdroppers are illegitimate users who are not registered. They are usually silent to hide their existence from the transmitter. On the other hand, although legitimate users are registered in the system, they may misbehave and eavesdrop the information signal of other legitimate receivers when they are idle. Thus, idle legitimate receivers are potential eavesdroppers. Yet, the frameworks proposed in [1], [3], [13]-[19] do not take into account the coexistence of passive eavesdroppers and potential eavesdroppers. In addition, these works do not fully utilize both artificial noise and energy signals for providing secure communication and improving wireless energy transfer in the system. Thus, the algorithms proposed in [1], [3], [13]-[19] may not be able to support the emerging QoS requirements on secure communication in systems with energy harvesting receivers, especially when passive eavesdroppers and potential eavesdroppers coexist in the environment.

In this paper, we address the above issues. To this end, the resource allocation algorithm design for secure communication in multiuser multiple-input single-output (MISO) systems with concurrent wireless information and power transfer is formulated as a non-convex optimization problem. The problem formulation encourages the dual use of both artificial noise and energy signals for facilitating efficient energy transfer and ensuring communication security. In particular, both artificial noise and energy signals are used to degrade the interception capabilities of the eavesdroppers when imperfect CSI of the potential eavesdroppers and no CSI of the passive eavesdroppers are available at the transmitter. For obtaining a tractable solution, we reformulate the considered problem by replacing a non-convex probabilistic constraint with a tractable convex deterministic constraint. The resulting reformulated non-convex optimization problem is solved by a semi-definite programming (SDP) based resource allocation algorithm. Fur- 
thermore, we propose a suboptimal resource allocation scheme which provides an excellent system performance at low computational complexity.

\section{SySTEM MODEL}

In this section, after introducing the notation used in this paper, we present the adopted multiuser downlink channel model for secure communication with simultaneous wireless information and power transfer.

\section{A. Notation}

For a square-matrix $\mathbf{X}, \operatorname{Tr}(\mathbf{X})$ denotes the trace of the matrix and $\mathbf{X} \succeq \mathbf{0}$ indicates that $\mathbf{X}$ is a positive semi-definite matrix. $(\mathbf{X})^{H}$ and $\operatorname{Rank}(\mathbf{X})$ denote the conjugate transpose and the rank of matrix $\mathbf{X}$, respectively. $[\mathbf{X}]_{a, b}$ extracts the $(a, b)$-th element of matrix $\mathbf{X}$. Matrix $\mathbf{I}_{N}$ denotes an $N \times N$ identity matrix. $\mathbb{C}^{N \times M}$ and $\mathbb{R}^{N \times M}$ denote the spaces of $N \times M$ matrices with complex and real entries, respectively. $\|\cdot\|$ and $|\cdot|$ denote the Euclidean norm of a matrix/vector and the absolute value of a complex scalar, respectively. $\operatorname{Re}(\cdot)$ extracts the real part of a complex-valued input. $\mathbb{H}^{N}$ represents the set of all $N$-by- $N$ complex Hermitian matrices. $\lambda_{\max }(\mathbf{X})$ and $\lambda_{j}(\mathbf{X})$ denote the maximum eigenvalue and the $j$-th eigenvalue of Hermitian matrix $\mathbf{X}$, respectively. The distribution of a circularly symmetric complex Gaussian (CSCG) vector with mean vector $\mathbf{x}$ and covariance matrix $\boldsymbol{\Sigma}$ is denoted by $\mathcal{C} \mathcal{N}(\mathbf{x}, \boldsymbol{\Sigma})$, and $\sim$ means "distributed as". For a real valued continuous function $f(\cdot), \nabla_{\mathbf{X}} f(\mathbf{X})$ represents the gradient of $f(\cdot)$ with respect to matrix $\mathbf{X} . \mathcal{E}\{\cdot\}$ represents statistical expectation.

\section{B. Downlink Channel Model}

We consider the downlink of a communication system which consists of a transmitter, $K$ legitimate receivers, and $J$ passive eavesdroppers. The transmitter is equipped with $N_{\mathrm{T}}$ transmit antennas while the legitimate receivers are single antenna devices and are able to decode information and harvest energy from radio signals, cf. Figure 1 Besides, the $J$ passive eavesdroppers are also equipped with a single antenna, respectively, and their goal is to decode the information transmitted by the transmitter without causing any interference to the communication channel. There are two types of legitimate receivers in the system; one desired receiver and $K-1$ idle receivers. In each scheduling slot, the transmitter conveys information to the desired receiver and transfers energy 11 to all legitimate receivers simultaneously. The $K-1$ idle receivers are supposed to harvest energy from the RF when they are inactive. However, the signals intended for the desired receiver may be overheard by the idle receivers since all the legitimate receivers are in the range of service coverage. If the idle receivers are malicious, they may eavesdrop the information signal of the desired receiver. Hence, the idle receivers are potential eavesdroppers which should be taken into account

\footnotetext{
${ }^{1}$ In this paper, a normalized energy unit, i.e., Joule-per-second, is adopted. Therefore, the terms power and energy are used interchangeably throughout this paper.
}

for providing secure communication. We focus on frequency flat communication channels. The downlink received signals at the desired receiver, idle receiver (potential eavesdropper) $k$, and passive eavesdroppen $j$ are given by, respectively,

$$
\begin{aligned}
y & =\mathbf{h}^{H} \mathbf{x}+z_{\mathrm{a}}, \\
y_{\text {Idle }, k} & =\mathbf{g}_{k}^{H} \mathbf{x}+z_{\mathrm{a}, k}, \forall k \in\{1, \ldots, K-1\}, \\
y_{\mathrm{E}, j} & =\mathbf{l}_{j}^{H} \mathbf{x}+z_{\mathrm{e}, j}, \forall j \in\{1, \ldots, J\},
\end{aligned}
$$

where $\mathbf{x} \in \mathbb{C}^{N_{\mathrm{T}} \times 1}$ denotes the transmitted symbol vector. $\mathbf{h}^{H} \in \mathbb{C}^{1 \times N_{\mathrm{T}}}$ is the channel vector between the transmitter and the desired receiver and $\mathrm{g}_{k}^{H} \in \mathbb{C}^{1 \times N_{\mathrm{T}}}$ is the channel vector between the transmitter and idle receiver (potential eavesdropper) $k \in\{1, \ldots, K-1\} . \mathbf{l}_{j}^{H} \in \mathbb{C}^{1 \times N_{\mathrm{T}}}$ is the channel vector between the transmitter and passive eavesdropper $j \in\{1, \ldots, J\}$. We note that variables, $\mathbf{h}, \mathbf{g}_{k}$, and $\mathbf{l}_{j}$ include the effects of the multipath fading, shadowing, and path loss of the associated channels. $z_{\mathrm{a}}, z_{\mathrm{a}, k}$, and $z_{\mathrm{e}, j}$ are thermal noises resulting from the receive antennas at the desired receiver, idle receiver $k$, and passive eavesdropper $j$, respectively. They are modeled as additive white Gaussian noises (AWGN) with zero mean and variance $\sigma_{\text {ant }}^{2}$, respectively, cf. Figure 1

\section{Hybrid Information and Energy Harvesting Receiver}

Different hardware circuitries [5], [20] are available for harvesting energy from the RF. The associated system models and the corresponding energy harvesting efficiencies can be significantly differen 3 . Besides, the signal used for decoding the modulated information cannot be reused for harvesting energy due to hardware limitations [5]. Therefore, we do not assume a particular type of energy harvesting circuit such that the resource allocation algorithm design is isolated from the specific hardware implementation details. In this paper, we adopt hybrid receivers [5], [7] which split the received signal into two power streams with power splitting ratios $1-\rho$ and $\rho$, cf. Figure 1 for harvesting energy and decoding the modulated information, respectively. Specifically, the power splitting unit is installed in the front-end of the receiver and is assumed to be a perfect passive analog device; it does not introduce any extra power gain, i.e., $0 \leq \rho \leq 1$, or noise. Indeed, the hybrid receiver is a generalization of traditional information receivers and energy harvesting receivers. In particular, by imposing power splitting ratios of $\rho=1$ and $\rho=0$, the hybrid receiver reduces to a traditional information receiver and an energy harvesting receiver, respectively. Moreover, the separated receiver proposed in the literature [2], [19] is also a special case of the considered hybrid receivers. Furthermore, we assume that a rechargeable battery is available at the receivers for storage of the harvested energy. The stored energy is preserved for future use to extend the lifetime of the receivers. We note that if the amount of harvested energy exceeds the maximum battery capacity, the excess harvested

\footnotetext{
${ }^{2}$ In practice, the number of passive eavesdroppers is not known at the transmitter. Nevertheless, by assuming a particular value for $J$, we can ensure that the transmitter is able to handle at most $J$ passive eavesdroppers.

${ }^{3}$ Designing hardware circuitries for harvesting energy from the RF is beyond the scope of this paper. Interested readers may refer to [5], [20] for further details.
} 


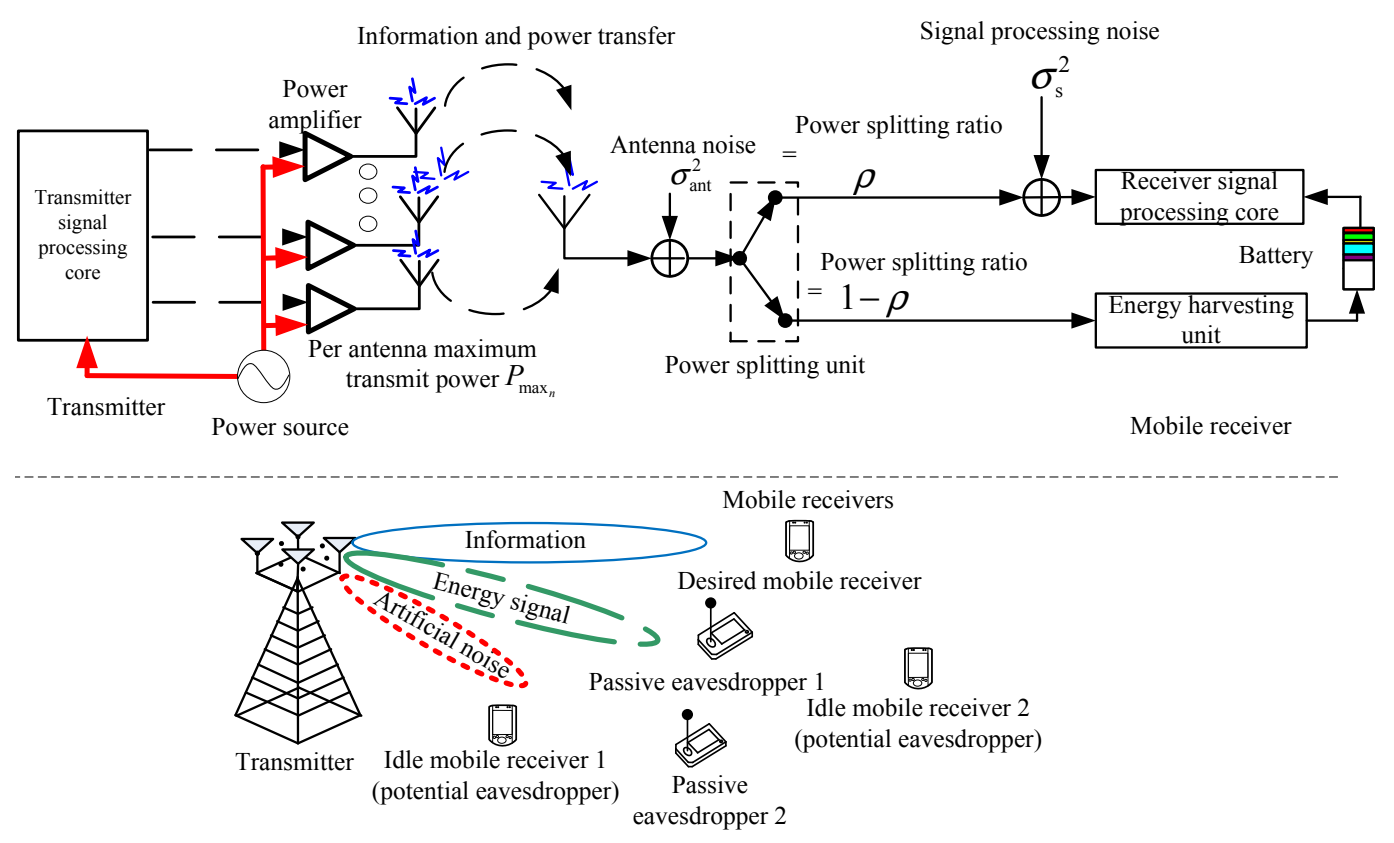

Fig. 1. Multiuser system model for $K=3$ legitimate mobile receivers and $J=2$ passive eavesdroppers. The upper half of the figure illustrates the block diagram of the transceiver model for wireless information and power transfer.

energy will be discarded. On the other hand, we assume that the desired information receiver has stored enough energy to decode the received signal.

\section{Channel State Information}

We assume a Time Division Duplex (TDD) system with slowly time-varying channels. At the beginning of each scheduling slot, the legitimate receivers send handshaking/beacon signal 4 to the transmitter to report their status (service requirements). The information embedded in the handshaking signals facilitates the downlink packet transmission. In particular, the downlink CSI of the transmitter-tolegitimate receiver channels is obtained through measuring the uplink pilots in the handshaking/beacon signals via channel reciprocity. Thus, the transmitter-to-legitimate receiver fading gains, $\mathbf{h}$ and $\mathbf{g}_{k}, \forall k \in\{1, \ldots, K-1\}$, can be reliably estimated at the transmitter at the beginning of each scheduling slot with negligible estimation errors. During the transmission, the desired receiver sends acknowledgement (ACK) packets to inform the transmitter of successful reception of information packets. As a result, the transmitter is able to refine the estimate of $\mathbf{h}$ frequently via the pilot sequences embedded in each ACK packet. Therefore, we can assume perfect CSI for the transmitter-to-desired receiver link during the whole transmission period. However, the CSI of the idle receivers becomes outdated during the transmission since there is no interaction between the transmitter and the idle receivers. To capture this effect, we use a deterministic model [21], [22] for modelling the resulting CSI uncertainty. The CSI of the link

\footnotetext{
${ }^{4}$ The legitimate receivers can either take turns to send the handshaking/beacon signals or transmit simultaneously with orthogonal pilot sequences.
}

between the transmitter and idle receiver $k$ is given by

$$
\begin{aligned}
\mathbf{g}_{k} & =\hat{\mathbf{g}}_{k}+\Delta \mathbf{g}_{k}, k \in\{1, \ldots, K-1\}, \text { and } \\
\Omega_{k} & \triangleq\left\{\Delta \mathbf{g}_{k} \in \mathbb{C}^{N_{\mathrm{T}} \times 1}: \Delta \mathbf{g}_{k}^{H} \Delta \mathbf{g}_{k} \leq \varepsilon_{k}^{2}\right\},
\end{aligned}
$$

where $\hat{\mathrm{g}}_{k} \in \mathbb{C}^{N_{\mathrm{T}} \times 1}$ is the channel estimate of idle receiver $k$ available at the transmitter at the beginning of a scheduling slot. $\Delta \mathrm{g}_{k}$ represents the unknown channel uncertainty of idle receiver $k$ due to the slow timing varying nature of the channel during transmission. For notational simplicity, we define a set $\Omega_{k}$ in (5) which contains all possible CSI uncertainties of idle receiver $k$. Specifically, the radius $\varepsilon_{k}>0$ represents the size of the uncertainty region of the estimated CSI of idle receiver $k$. In practice, the value of $\varepsilon_{k}^{2}$ depends on the coherence time of the associated channel and the time between channel estimation and packet transmission.

On the other hand, the passive eavesdroppers, which are also present in the system, are usually silent to hide their existence from the transmitter. As a result, the CSI of the passive eavesdroppers cannot be measured at the transmitter based on handshaking signals. To facilitate the resource allocation algorithm design, we assume that the transmitter knows that the eavesdroppers are equipped with single antennas. In addition, except for the variance, the distribution of the channels between the transmitter and the passive eavesdroppers is assumed to be known at the transmitter.

\section{E. Artificial Noise and Energy Signal Generation}

In order to provide secure communication and to facilitate energy harvesting at the desired receivers, artificial noise signals and energy signals are generated at the transmitter. In particular, both signals are able to degrade the channels between the transmitter and the passive eavesdroppers and 
act as energy source for energy harvesting. The transmitter chooses the transmit signal vector $\mathbf{x}$ as

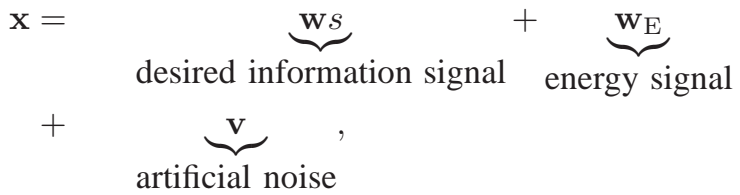

where $s \in \mathbb{C}$ and $\mathbf{w} \in \mathbb{C}^{N_{\mathrm{T}} \times 1}$ are the information-bearing signal and the corresponding beamforming vector for the desired receiver, respectively. We assume without loss of generally that $\mathcal{E}\left\{|s|^{2}\right\}=1$. $\mathbf{v} \in \mathbb{C}^{N_{\mathrm{T}} \times 1}$ is the artificial noise vector generated by the transmitter to combat both potential and passive eavesdroppers. $\mathbf{v}$ is modeled as a complex Gaussian random vector with

$$
\mathbf{v} \sim \mathcal{C N}(\mathbf{0}, \mathbf{V})
$$

where $\mathbf{V} \in \mathbb{H}^{N_{\mathrm{T}}}, \mathbf{V} \succeq \mathbf{0}$, denotes the covariance matrix of the artificial noise. The artificial noise signal $\mathbf{v}$ is unknown to both the legitimate receivers and passive eavesdroppers. On the other hand, $\mathbf{w}_{\mathrm{E}}$ is a Gaussian pseudo-random sequence 5 which is used to facilitate efficient energy transfer and is known to all legitimate (both active and idle) receivers. $\mathbf{w}_{\mathrm{E}}$ is modeled as a complex Gaussian pseudo-random vector with

$$
\mathbf{w}_{\mathrm{E}} \sim \mathcal{C N}\left(\mathbf{0}, \mathbf{W}_{\mathrm{E}}\right),
$$

where $\mathbf{W}_{\mathrm{E}} \in \mathbb{H}^{N_{\mathrm{T}}}, \mathbf{W}_{\mathrm{E}} \succeq \mathbf{0}$ denotes the covariance matrix of the pseudo-random energy signal.

Remark 1: We assume that energy signal $\mathbf{w}_{\mathrm{E}}$ is a pseudorandom signal which is perfectly known at the transmitter and the desired receivers but not at the passive eavesdroppers. This can be realized by using a short secret key as seed information for the pseudo-random sequence generator used for generating the energy signal sequences, where the transmitter regularly changes the seeds to prevent the sequence from being cracked by the passive eavesdroppers. The seeds information used at the transmitter can be delivered to the desired receivers securely by exploiting e.g. the reciprocity of the channels between the transmitter and the legitimated receivers [23].

Remark 2: We note that unlike other system models adopted in the literature, e.g. [13]-[15], the artificial noise and energy signals in the considered system can be exploited by the legitimate receivers. Although the transmitter could also solely use the energy of the information carrying signal for supplying energy to the legitimate receivers [5], [7], increasing the transmit power of the information signal for facilitating energy harvesting increases the susceptibility to eavesdropping. As a result, in this paper, we advocate the dual use of artificial noise signals and energy signals in providing simultaneous security and efficient energy transfer.

\section{Resource Allocation Algorithm Design}

In this section, we first define the channel capacity and secrecy capacity as the system performance metrics. Then,

\footnotetext{
${ }^{5}$ For energy transfer, the energy sequence is not required to be generated by a Gaussian pseudo-random source. However, in this paper, a Gaussian pseudo-random energy sequence is assumed to be able to also provide secure communication.
}

we formulate the corresponding resource allocation algorithm design as an optimization problem. For the sake of notational simplicity, we define the following variables: $\mathbf{H}=\mathbf{h h}^{H}$, $\mathbf{G}_{k}=\mathbf{g}_{k} \mathbf{g}_{k}^{H}, k \in\{1, \ldots, K-1\}$, and $\mathbf{L}_{j}=\mathbf{l}_{j} \mathbf{l}_{j}^{H}, j \in$ $\{1, \ldots, J\}$.

\section{A. System Capacity and Secrecy Capacity}

The energy signal $\mathbf{w}_{\mathrm{E}}$ is a Gaussian pseudo-random sequence which is only known at the legitimate receivers. Hence, interference cancellation can be performed at the legitimated receivers for improving the channel capacity for information decoding. As a result, given perfect CSI at the receiver, the channel capacity (bit/s/Hz) between the transmitter and the desired receiver can be rewritten as

$$
\begin{aligned}
C^{\mathrm{IC}} & =\log _{2}\left(1+\Gamma^{\mathrm{IC}}\right) \text { and } \\
\Gamma^{\mathrm{IC}} & =\frac{\rho \mathbf{w}^{H} \mathbf{H w}}{\rho\left(\sigma_{\text {ant }}^{2}+\operatorname{Tr}(\mathbf{H V})\right)+\sigma_{\mathrm{s}}^{2}},
\end{aligned}
$$

where $\Gamma^{\mathrm{IC}}$ is the received signal-to-interference-plus-noise ratio (SINR) at the desired receiver when interference cancellation is performed [8], i.e., $\operatorname{Tr}\left(\mathbf{H} \mathbf{W}_{\mathrm{E}}\right)$ has been removed. $\sigma_{\mathrm{s}}^{2}$ is the signal processing noise power at the receiven, $\mathrm{cf}$. Figure 11 On the other hand, interference cancellation can also be performed at the idle receivers. Therefore, the channel capacity between the transmitter and idle receiver (potential eavesdropper) $k$ is given by

$$
\begin{aligned}
C_{\mathrm{I}_{k}}^{\mathrm{IC}} & =\log _{2}\left(1+\Gamma_{k}^{\mathrm{IC}}\right) \text { and } \\
\Gamma_{\mathrm{I}_{k}}^{\mathrm{IC}} & =\frac{\rho_{k} \mathbf{w}^{H} \mathbf{G}_{k} \mathbf{w}}{\rho_{k}\left(\sigma_{\mathrm{ant}}^{2}+\operatorname{Tr}\left(\mathbf{G}_{k} \mathbf{V}\right)\right)+\sigma_{\mathrm{s}}^{2}} \\
& \stackrel{(a)}{\leq} \frac{\mathbf{w}^{H} \mathbf{G}_{k} \mathbf{w}}{\sigma_{\mathrm{ant}}^{2}+\operatorname{Tr}\left(\mathbf{G}_{k} \mathbf{V}\right)+\sigma_{\mathrm{s}}^{2}},
\end{aligned}
$$

where $\rho_{k}$ and $\Gamma_{\mathrm{I}_{k}}^{\mathrm{IC}}$ are the power splitting ratio and the received SINR at idle receiver $k$, respectively. $(a)$ is due to the fact that $\Gamma_{\mathrm{I}_{k}}^{\mathrm{IC}}$ is a monotonically increasing function of $\rho_{k}$. The physical meaning of 11] is that idle receiver $k$ gives up the opportunity to harvest energy and devotes all the received power to eavesdropping.

In practice, the transmitter does not know the location of the passive eavesdroppers. As a result, we design the resource allocation algorithm assuming an unfavourable scenario for the location of the passive eavesdroppers. In particular, we assume that passive eavesdropper $j$ is close to the transmitter and is located at the reference distance of the path loss mode 7 . Thus, under this scenario, the capacity between the transmitter and

\footnotetext{
${ }^{6} \mathrm{We}$ assume that the signal processing and thermal noise characteristics are identical for all receivers due to similar hardware architectures.

${ }^{7}$ In practice, the reference distance can be treated as a security guard zone and we assume it is known that passive eavesdroppers do not exist inside this zone.
} 
passive eavesdropper $j \in\{1, \ldots, J\}$ is given by

$$
\begin{aligned}
C_{\mathrm{PE}_{j}} & \stackrel{(a)}{=} \log _{2}\left(1+\Gamma_{\mathrm{PE}_{j}}\right) \text { and } \\
\Gamma_{\mathrm{PE}_{j}} & =\frac{\mathbf{w}^{H} \mathbf{L}_{j} \mathbf{w}}{\operatorname{Tr}\left(\mathbf{L}_{j} \mathbf{W}_{\mathrm{E}}\right)+\operatorname{Tr}\left(\mathbf{L}_{j} \mathbf{V}\right)+\sigma_{\text {ant }}^{2}+\sigma_{\mathrm{s}}^{2}} \\
& \stackrel{(b)}{\leq} \frac{\mathbf{w}^{H} \mathbf{L}_{j}^{\mathrm{UP}} \mathbf{w}}{\operatorname{Tr}\left(\mathbf{L}_{j}^{\mathrm{UP}} \mathbf{W}_{\mathrm{E}}\right)+\operatorname{Tr}\left(\mathbf{L}_{j}^{\mathrm{UP}} \mathbf{V}\right)+\sigma_{\text {ant }}^{2}+\sigma_{\mathrm{s}}^{2}}\left(\tilde{\mathbf{w}}^{H} \tilde{\mathbf{L}}_{j} \mathbf{w}\right. \\
& \stackrel{(c)}{=} \frac{\operatorname{Tr}\left(\tilde{\mathbf{L}}_{j} \mathbf{V}\right)+\tilde{\sigma}_{j}^{2}}{\operatorname{Tr}\left(\tilde{\mathbf{L}}_{j} \mathbf{W}_{\mathrm{E}}\right)+\operatorname{Tr}}
\end{aligned}
$$

where $\mathbf{L}_{j}^{\mathrm{UP}}=\mathbf{l}_{j}^{\mathrm{UP}}\left(\mathbf{l}_{j}^{\mathrm{UP}}\right)^{H}$, and $\mathbf{l}_{j}^{\mathrm{UP}}$ is the channel vector between the transmitter and a passive eavesdropper located at the reference distance. We note that the elements of $\mathrm{l}_{j}^{\mathrm{UP}}$ and $\mathbf{l}_{j}$ capture the joint effect of small scale fading and shadowing in the same manner. Yet, the path loss coefficient contained in $\mathrm{l}_{j}^{\mathrm{UP}}$ is calculated at the reference distance which results in $\left\|\mathbf{l}_{j}^{\mathrm{UP}}\right\| \geq\left\|\mathbf{1}_{j}\right\|$. The equality in $(a)$ is due to the assumption that the energy signal $\mathbf{w}_{\mathrm{E}}$ is a Gaussian random signal. The inequality in $(b)$ is due to the fact that the SINR in 13 is a monotonically increasing function with respect to $\mathbf{L}_{j}$ and $\left\|\mathbf{l}_{j}^{\mathrm{UP}}\right\| \geq\left\|\mathbf{l}_{j}\right\|$. On the other hand, $\tilde{\mathbf{L}}_{j}=\tilde{\mathbf{l}}_{j} \tilde{\mathbf{l}}_{j}^{H}=\frac{\mathbf{L}_{j}^{\mathrm{UP}}}{\mathcal{E}\left\{\left\|\mathbf{l}_{j}^{\mathrm{UP}}\right\|^{2}\right\}}$ and $\tilde{\sigma}_{j}^{2}=\frac{\sigma_{\text {ant }}^{2}+\sigma_{s}^{2}}{\mathcal{E}\left\{\left\|\mathbf{1}_{j}^{\mathrm{UP}}\right\|^{2}\right\}}$ in $(c)$ denote the normalized channel matrix and noise power, respectively. Specifically, $(c)$ in 14 is obtained by dividing 8 both the nominator and denominator of the upper bound SINR by $\mathcal{E}\left\{\left\|\mathbf{l}_{j}^{\mathrm{UP}}\right\|^{2}\right\}$. Besides, since $\mathcal{E}\left\{\left\|\mathbf{1}_{1}^{\mathrm{UP}}\right\|^{2}\right\}=\mathcal{E}\left\{\left\|\mathbf{1}_{2}^{\mathrm{UP}}\right\|^{2}\right\}=\ldots=\mathcal{E}\left\{\left\|\mathbf{1}_{J}^{\mathrm{UP}}\right\|^{2}\right\}$, we rewrite $\tilde{\sigma}_{j}^{2}$ as $\tilde{\sigma}^{2}$ in the sequel without loss of generality. We note that the passive eavesdroppers are unable to perform interference cancellation to remove $\operatorname{Tr}\left(\mathbf{L}_{j} \mathbf{W}_{\mathrm{E}}\right)$ since the energy sequence $\mathbf{w}_{\mathrm{E}}$ is only known at the legitimate receivers. With a slight abuse of notation, we reuse variables $C_{\mathrm{I}_{k}}^{\mathrm{IC}}$ and $C_{\mathrm{PE}_{j}}$ to denote their upper bounds by replacing the SINRs $\Gamma_{I_{h}}^{\mathrm{IC}}$ and $\Gamma_{\mathrm{PE}_{j}}$ in (10) and 12 with their upper bounds in (11) and (14), respectively.

Therefore, the maximum achievable secrecy capacity between the transmitter and the desired receiver can be expressed as [13]

$$
\begin{aligned}
& C_{\mathrm{sec}} \\
= & {\left[C^{\mathrm{IC}}-\max \left\{\max _{k \in\{1, \ldots, K-1\}} C_{\mathrm{I}_{k}}^{\mathrm{IC}}, \max _{j \in\{1, \ldots, J\}} C_{\mathrm{PE}_{j}}\right\}\right]^{+} }
\end{aligned}
$$

$C_{\text {sec }}$ quantifies the maximum achievable data rate at which a transmitter can reliably send secret information to the desired receiver such that the eavesdroppers are unable to decode the received signal even if the eavesdroppers have infinite computational power for decoding the received signals.

Remark 3: Equations (10)-14) reveal that the artificial noise signal, $\mathbf{v}$, and the energy signal, $\mathbf{w}_{\mathrm{E}}$, have different effectiveness in providing communication security. In particular, the artificial noise signal $\mathbf{v}$ is able to degrade the channels of both idle receivers (potential eavesdroppers) and passive eavesdroppers simultaneously, while $\mathbf{w}_{\mathrm{E}}$ can only degrade the channels of the passive eavesdroppers. On the other hand, both

\footnotetext{
${ }^{8}$ The normalization facilitates the resource allocation algorithm design in the later parts of this paper.
}

$\mathbf{v}$ and $\mathbf{w}_{\mathrm{E}}$ have the same effectiveness in facilitating energy transfer to the legitimate receivers.

\section{B. Optimization Problem Formulation}

The optimal resource allocation policy, $\left\{\mathbf{w}^{*}, \rho^{*}, \mathbf{W}_{\mathrm{E}}^{*}, \mathbf{V}^{*}\right\}$, for minimizing the total power radiated by the transmitter, can be obtained by solving

$$
\begin{aligned}
& \underset{\mathbf{W}_{\mathrm{E}}, \mathbf{V} \in \mathbb{H}^{N_{\mathrm{T}}, \mathbf{w}, \rho}}{\operatorname{minimize}}\|\mathbf{w}\|^{2}+\operatorname{Tr}(\mathbf{V})+\operatorname{Tr}\left(\mathbf{W}_{\mathrm{E}}\right) \\
& \text { s.t. } \quad \mathbf{C} 1: \frac{\rho\left|\mathbf{h}^{H} \mathbf{w}\right|^{2}}{\rho\left(\sigma_{\text {ant }}^{2}+\operatorname{Tr}(\mathbf{H V})\right)+\sigma_{\mathrm{s}}^{2}} \geq \Gamma_{\text {req }} \text {, } \\
& \text { C2: } \max _{\Delta \mathbf{g}_{k} \in \Omega_{k}} \frac{\left|\mathbf{g}_{k}^{H} \mathbf{w}\right|^{2}}{\sigma_{\text {ant }}^{2}+\operatorname{Tr}\left(\mathbf{G}_{k} \mathbf{V}\right)+\sigma_{\mathrm{s}}^{2}} \leq \Gamma_{\mathrm{tol}_{k}}, \forall k, \\
& \text { C3: } \operatorname{Pr}\left(\max _{j \in\{1, \ldots, J\}}\left\{\frac{\mathbf{w}^{H} \tilde{\mathbf{L}}_{j} \mathbf{w}}{\operatorname{Tr}\left(\tilde{\mathbf{L}}_{j} \mathbf{W}_{\mathrm{E}}\right)+\operatorname{Tr}\left(\tilde{\mathbf{L}}_{j} \mathbf{V}\right)+\tilde{\sigma}_{j}^{2}}\right\} \leq \Gamma_{\text {tol }}\right) \\
& \geq \kappa, \\
& \text { C4: }(1-\rho) \eta\left|\mathbf{h}^{H} \mathbf{w}\right|^{2}+(1-\rho) \eta(\operatorname{Tr}(\mathbf{H V}) \\
& \left.+\operatorname{Tr}\left(\mathbf{H} \mathbf{W}_{\mathrm{E}}\right)+\sigma_{\text {ant }}^{2}\right) \geq P_{\min }, \\
& \text { C5: } \min _{\Delta \mathbf{g}_{k} \in \Omega_{k}} \eta\left|\mathbf{g}_{k}^{H} \mathbf{w}\right|^{2}+\eta\left(\operatorname{Tr}\left(\mathbf{G}_{k} \mathbf{V}\right)\right. \\
& \left.+\operatorname{Tr}\left(\mathbf{G}_{k} \mathbf{W}_{\mathrm{E}}\right)+\sigma_{\text {ant }}^{2}\right) \geq P_{\min _{k}}, \forall k, \\
& \text { C6: }\left[\mathbf{w w}^{H}\right]_{n, n}+[\mathbf{V}]_{n, n}+\left[\mathbf{W}_{\mathrm{E}}\right]_{n, n} \\
& \leq P_{\max _{n}}, \forall n \in\left\{1, \ldots, N_{\mathrm{T}}\right\} \\
& \text { C7: } 0 \leq \rho \leq 1, \quad \text { C8: V, } \mathbf{W}_{\mathrm{E}} \succeq \mathbf{0} .
\end{aligned}
$$

In $\mathrm{C} 1, \Gamma_{\text {req }}$ denotes the minimum SINR of the desired receiver required for information decoding. This constraint guarantees that the channel capacity between the transmitter and the desired receiver is $C^{\mathrm{IC}} \geq \log _{2}\left(1+\Gamma_{\text {req }}\right)$. Constraint $\mathrm{C} 2$ is imposed such that for a given CSI uncertainty set $\Omega_{k}$, the maximum received SINR at idle receiver (potential eavesdropper) $k$ is less than the maximum tolerable received SINR $\Gamma_{\text {tol }_{k}}$. In practice, the transmitter sets $\Gamma_{\text {req }} \gg \Gamma_{\text {tol }_{k}}>0, \forall k \in$ $\{1, \ldots, K-1\}$, to ensure secure communication. Specifically, if the above optimization problem is feasible and passive eavesdroppers do not exist in the system, the adopted problem formulation guarantees that the secrecy capacity is bounded below by $C_{\text {sec }} \geq \log _{2}\left(1+\Gamma_{\text {req }}\right)-\log _{2}\left(1+\max _{k}\left\{\Gamma_{\text {tol }_{k}}\right\}\right) \geq 0$. We note that although $\Gamma_{\text {req }}$ and $\Gamma_{\text {tol }_{k}}$ in $\mathrm{C} 1$ and $\mathrm{C} 2$, respectively, are not optimization variables in this paper, a balance between secrecy capacity and system capacity can be struck by varying their values. In $\mathrm{C} 3, \Gamma_{\text {tol }}$ represents the maximum received SINR tolerance for successfully decoding at passive eavesdropper $j$. This constraint specifies the minimum outage requirement at all passive eavesdroppers. In particular, the maximum received SINR among all passive eavesdroppers is required to be smaller than the maximum tolerable received SINR9 $\Gamma_{\text {tol }}$ with at least probability $\kappa$. For instance, if $\kappa=0.99, \Gamma_{\text {req }} \geq \Gamma_{\text {tol }}$, and an idle receiver does not exist

\footnotetext{
${ }^{9}$ In fact, the system operator can adjust the values of the maximum received SINR tolerance $\Gamma_{\text {tol }}$ to account for performance variations due to potential system model mismatches such as the assumption that $\mathbf{w}_{\mathrm{E}}$ is Gaussian distributed.
} 
in the system, constraints $\mathrm{C} 1$ and $\mathrm{C} 3$ together guarantee that the secrecy capacity between the transmitter and the desired receiver is bounded below by $C_{\text {sec }}=\log _{2}\left(1+\Gamma_{\text {req }}\right)-$ $\log _{2}\left(1+\max _{j}\left\{\Gamma_{\mathrm{PE}_{j}}\right\}\right) \geq \log _{2}\left(1+\Gamma_{\text {req }}\right)-\log _{2}\left(1+\Gamma_{\text {tol }}\right)$ with probability 0.99 . We note that the proposed problem formulation and resource allocation schemes are also valid for $\kappa=0$. Specifically, for $\kappa=0$ and continuous random variables $\Gamma_{\mathrm{PE}_{j}} \in(0, \infty)$, constraint $\mathrm{C} 3$ is always satisfied. Hence, for $\kappa=0$, constraint $\mathrm{C} 3$ can be safely removed from the optimization problem formulation without loss of optimality. Besides, although the number of eavesdroppers $J$ is not known at the transmitter, $J$ in $\mathrm{C} 3$ represents the maximum tolerable number of passive eavesdroppers that the transmitter can handle. Furthermore, we note that we do not maximize the secrecy capacity in this paper as this would not necessarily lead to a power efficient resource allocation. $P_{\min }$ and $P_{\min _{k}}$ in $\mathrm{C} 4$ and $\mathrm{C} 5$ set the minimum required power transfer to the desired information receiver and idle receiver $k$, respectively. We note that for given CSI uncertainty sets $\Omega_{k}, \forall k$, the transmitter can only guarantee the minimum required power transfer to the $K-1$ idle receivers if they use all their received power for energy harvesting, i.e., the idle receivers do not intend to eavesdrop. $\eta$ in C4 and C5 denotes the energy harvesting efficiency of the receivers in converting the received radio signal to electrical energy. Furthermore, in practice, each transmit antenna has its own power amplifier in its analog front-end in practice, cf. Figure 1 . Hence, the power radiated by each antenna is limited by the maximum transmit power of each power amplifier. In C6, we take this physical limitation into account by limiting the maximum transmit power from antenna $n$ to $P_{\max _{n}}$. We note that the introduction of a joint power consumption constraint for all power amplifiers is not necessary as long as $\sum_{n=1}^{N_{\mathrm{T}}} P_{\max _{n}} \leq P_{\mathrm{PG}}$, where $P_{\mathrm{PG}}$ is the maximum power supply at the transmitter. $\mathrm{C} 7$ is the boundary constraint for the power splitting variable $\rho . \mathrm{C} 8$ and $\mathbf{V}, \mathbf{W}_{\mathrm{E}} \in \mathbb{H}^{N_{\mathrm{T}}}$ constrain matrices $\mathbf{V}$ and $\mathbf{W}_{\mathrm{E}}$ to be positive semi-definite Hermitian matrices such that both are valid covariance matrices.

Remark 4: We would like to emphasize that the considered problem formulation is a generalization of the cases where only artificial noise or only energy signal allocation are performed. If $\mathbf{w}_{\mathrm{E}}$ is not known at the legitimate receivers, it cannot be cancelled at the legitimate receivers. In this case, we can set $\mathbf{w}_{\mathrm{E}}=\mathbf{0}$ in the problem formulation without loss of optimality. This is because if $\mathbf{w}_{\mathrm{E}} \neq \mathbf{0}$ and interference cancellation cannot be performed, we can define a new resource allocation policy with a new artificial noise signal, $\mathbf{v}_{\text {new }}=\mathbf{v}+\mathbf{w}_{\mathrm{E}}$, and a new energy signal, $\mathbf{w}_{\mathrm{E}_{\text {new }}}=\mathbf{0}$, such that the new resource allocation policy consumes the same amount of energy as the original solution and satisfies all the constraints.

\section{Solution of the Optimization Problem}

The optimization problem in (16) is a non-convex quadratically constrained quadratic program (QCQP) which involves chance constrained programming [24], [25] and semi-infinite programming. In particular, the non-convexity with respect to the beamforming vector $\mathbf{w}$ for the information signal and the power splitting ratio $\rho$ is due to constraints $\mathrm{C} 1$ and $\mathrm{C} 4$. Besides, constraints $\mathrm{C} 2$ and $\mathrm{C} 5$ involve infinitely many inequality constraints due to the continuity of the CSI uncertainty sets. Furthermore, the probabilistic (chance) constraint C3 couples all optimization variables and is non-convex. In general, even if we remove chance constraint $\mathrm{C} 3$, there is no standard approach for solving non-convex optimization problems. In some extreme cases, an exhaustive search approach is required to obtain a globally optimal solution which is computationally intractable even for a moderate system size. In order to derive an efficient resource allocation algorithm for the considered problem, we first recast the original problem as a semidefinite programming (SDP) problem in order to avoid the non-convexity associated with constraints $\mathrm{C} 1$ and $\mathrm{C} 4$. Then, we convert the infinite number of constraints in $\mathrm{C} 2$ and $\mathrm{C} 5$ into an equivalent finite number of constraints. Subsequently, we propose a tractable convex constraint as a replacement for non-convex constraint $\mathrm{C} 3$. The reformulated problem with constraint replacement serves as a performance lower bound for the original problem formulation. Finally, we use semidefinite programming relaxation (SDR) [26], [27] to obtain the optimal resource allocation solution for the reformulated problem. In practice, the considered problem may be infeasible when the QoS requirements are stringent and/or the channels are in unfavourable conditions. However, in the sequel, we assume that the problem is always feasible for studying the design of different resource allocation schemes.

\section{A. Semi-definite Programming Relaxation}

To facilitate SDP relaxation, we define $\mathbf{W}=\mathbf{w} \mathbf{w}^{H}$ and rewrite problem in terms of $\mathbf{W}$ as

$$
\begin{aligned}
& \underset{\mathbf{W}, \mathbf{V}, \mathbf{W}_{\mathrm{E}} \in \mathbb{H}^{N_{\mathrm{T}}, \rho}}{\operatorname{minimize}} \operatorname{Tr}(\mathbf{W})+\operatorname{Tr}(\mathbf{V})+\operatorname{Tr}\left(\mathbf{W}_{\mathrm{E}}\right) \\
& \text { s.t. C1: } \frac{\operatorname{Tr}(\mathbf{H W})}{\sigma_{\text {ant }}^{2}+\operatorname{Tr}(\mathbf{H V})+\frac{\sigma_{\mathrm{s}}^{2}}{\rho}} \geq \Gamma_{\text {req }} \text {, } \\
& \text { C2: } \max _{\Delta \mathbf{g}_{k} \in \Omega_{k}} \frac{\operatorname{Tr}\left(\mathbf{G}_{k} \mathbf{W}\right)}{\sigma_{\text {ant }}^{2}+\operatorname{Tr}\left(\mathbf{G}_{k} \mathbf{V}\right)+\sigma_{\mathrm{s}}^{2}} \leq \Gamma_{\text {tol }_{k}}, \forall k, \\
& \text { C3: } \operatorname{Pr}\left(\max _{j \in\{1, \ldots, J\}}\left\{\frac{\operatorname{Tr}\left(\tilde{\mathbf{L}}_{j} \mathbf{W}\right)}{\operatorname{Tr}\left(\tilde{\mathbf{L}}_{j} \mathbf{W}_{\mathrm{E}}\right)+\operatorname{Tr}\left(\tilde{\mathbf{L}}_{j} \mathbf{V}\right)+\tilde{\sigma}_{j}^{2}}\right\} \leq \Gamma_{\text {tol }}\right) \\
& \geq \kappa, \\
& \text { C4: } \operatorname{Tr}(\mathbf{H W})+\operatorname{Tr}\left(\mathbf{H W} \mathbf{E}_{\mathrm{E}}\right)+\operatorname{Tr}(\mathbf{H V}) \\
& +\sigma_{\text {ant }}^{2} \geq \frac{P_{\min }}{(1-\rho) \eta}, \\
& \text { C5: } \min _{\Delta \mathbf{g}_{k} \in \Omega_{k}} \operatorname{Tr}\left(\mathbf{G}_{k} \mathbf{W}\right)+\operatorname{Tr}\left(\mathbf{G}_{k} \mathbf{V}\right)+\operatorname{Tr}\left(\mathbf{G}_{k} \mathbf{W}_{\mathrm{E}}\right) \\
& +\sigma_{\text {ant }}^{2} \geq \frac{P_{\min _{k}}}{\eta}, \forall k \\
& \text { C6: } \operatorname{Tr}\left(\boldsymbol{\Psi}_{n}\left(\mathbf{W}+\mathbf{V}+\mathbf{W}_{\mathrm{E}}\right)\right) \leq P_{\max _{n}}, \forall n \in\left\{1, \ldots, N_{\mathrm{T}}\right\} \text {, } \\
& \mathrm{C} 7: 0 \leq \rho \leq 1, \mathbf{C} 8: \mathbf{W} \succeq \mathbf{0}, \mathbf{V} \succeq \mathbf{0}, \mathbf{W}_{\mathrm{E}} \succeq \mathbf{0}, \\
& \text { C9: } \operatorname{Rank}(\mathbf{W})=1 \text {, }
\end{aligned}
$$

where $\mathbf{W} \succeq \mathbf{0}, \mathbf{W} \in \mathbb{H}^{N_{\mathrm{T}}}$, and $\operatorname{Rank}(\mathbf{W})=1$ in 17 are imposed to guarantee that $\mathbf{W}=\mathbf{w} \mathbf{w}^{H}$ holds after optimizing $\mathbf{W}$. We note that the per-antenna radiated power in constraint 
C6 in (16) can be represented as $\operatorname{Tr}\left(\mathbf{\Psi}_{n}\left(\mathbf{W}+\mathbf{V}+\mathbf{W}_{\mathrm{E}}\right)\right)$, where $\boldsymbol{\Psi}_{n}=\mathbf{e}_{n} \mathbf{e}_{n}^{H}$ and $\mathbf{e}_{n} \in \mathbb{R}^{N_{\mathrm{T}} \times 1}$ is the $n$-th unit vector of length $N_{\text {T }}$, i.e., $\left[\mathbf{e}_{n}\right]_{n, 1}=1$ and $\left[\mathbf{e}_{n}\right]_{b, 1}=0, \forall b \neq n, b \in$ $\left\{1, \ldots, N_{\mathrm{T}}\right\}$. After variable transformation $\mathbf{W}=\mathbf{w w}^{H}$ and some manipulations, it can be observed that constraints $\mathrm{C} 1$ and $\mathbf{C} 4$ are convex with respect to $\left\{\mathbf{W}, \mathbf{V}, \mathbf{W}_{\mathrm{E}}, \rho\right\}$. Next, we handle constraints $\mathrm{C} 2$ and $\mathrm{C} 5$. Although constraints $\mathrm{C} 2$ and C5 are convex with respect to the optimization variables (after some mathematical manipulations), they are semi-infinite constraints which are generally intractable for resource allocation algorithm design. To facilitate the solution, we transform constraints C2 and C5 into linear matrix inequalities (LMIs) using the following lemma:

Lemma 1 (S-Procedure [28]): Let a function $f_{m}(\mathbf{x}), m \in$ $\{1,2\}, \mathbf{x} \in \mathbb{C}^{N \times 1}$, be defined as

$$
f_{m}(\mathbf{x})=\mathbf{x}^{H} \mathbf{A}_{m} \mathbf{x}+2 \operatorname{Re}\left\{\mathbf{b}_{m}^{H} \mathbf{x}\right\}+c_{m},
$$

where $\mathbf{A}_{m} \in \mathbb{H}^{N}, \mathbf{b}_{m} \in \mathbb{C}^{N \times 1}$, and $c_{m} \in \mathbb{R}^{1 \times 1}$. Then, the implication $f_{1}(\mathbf{x}) \leq 0 \Rightarrow f_{2}(\mathbf{x}) \leq 0$ holds if and only if there exists a $\delta \geq 0$ such that

$$
\delta\left[\begin{array}{cc}
\mathbf{A}_{1} & \mathbf{b}_{1} \\
\mathbf{b}_{1}^{H} & c_{1}
\end{array}\right]-\left[\begin{array}{cc}
\mathbf{A}_{2} & \mathbf{b}_{2} \\
\mathbf{b}_{2}^{H} & c_{2}
\end{array}\right] \succeq \mathbf{0},
$$

provided that there exists a point $\hat{\mathbf{x}}$ such that $f_{k}(\hat{\mathbf{x}})<0$.

As a result, we can apply Lemma 1 to constraint $\mathrm{C} 2$. In particular, we substitute $\mathbf{g}_{k}=\hat{\mathbf{g}}_{k}+\Delta \mathbf{g}_{k}$ in constraint $\mathrm{C} 2$. Therefore, the implication

$$
\begin{aligned}
& \Delta \mathbf{g}_{k}^{H} \Delta \mathbf{g}_{k} \leq \varepsilon_{k}^{2} \\
\Rightarrow & 0 \geq \Delta \mathbf{g}_{k}^{H}\left(\frac{\mathbf{W}}{\Gamma_{\mathrm{tol}_{k}}}-\mathbf{V}\right) \Delta \mathbf{g}_{k}+2 \operatorname{Re}\left\{\hat{\mathbf{g}}_{k}^{H}\left(\frac{\mathbf{W}}{\Gamma_{\mathrm{tol}_{k}}}-\mathbf{V}\right) \Delta \mathbf{g}_{k}\right\} \\
& +\hat{\mathbf{g}}_{k}^{H}\left(\frac{\mathbf{W}}{\Gamma_{\mathrm{tol}_{k}}}-\mathbf{V}\right) \hat{\mathbf{g}}_{k}-\sigma_{\text {ant }}^{2}-\sigma_{\mathrm{s}}^{2}, \forall k,
\end{aligned}
$$

holds if and only if there exist $\delta_{k} \geq 0, k \in\{1, \ldots, K-1\}$, such that the following LMI constraints hold:

$$
\begin{aligned}
& \mathrm{C} 2: \mathbf{S}_{\mathrm{C}_{2_{k}}}\left(\mathbf{W}, \mathbf{V}, \delta_{k}\right) \\
& =\left[\begin{array}{cc}
\delta_{k} \mathbf{I}_{N_{T}}+\mathbf{V} & \mathbf{V} \hat{\mathbf{g}}_{k} \\
\hat{\mathbf{g}}_{k}^{H} \mathbf{V} & -\delta_{k} \varepsilon_{k}^{2}+\sigma_{\text {ant }}^{2}+\sigma_{\mathrm{s}}^{2}+\hat{\mathbf{g}}_{k}^{H} \mathbf{V} \hat{\mathbf{g}}_{k}
\end{array}\right] \\
& -\quad \frac{1}{\Gamma_{\mathrm{tol}_{k}}} \mathbf{U}_{\mathbf{g}_{k}}^{H} \mathbf{W} \mathbf{U}_{\mathbf{g}_{k}} \succeq \mathbf{0}, \forall k,
\end{aligned}
$$

where $\mathbf{U}_{\mathbf{g}_{k}}=\left[\begin{array}{ll}\mathbf{I}_{N_{\mathrm{T}}} & \hat{\mathbf{g}}_{k}\end{array}\right]$. Similarly, by using Lemma 1, constraint $\mathrm{C} 5$ can be equivalently written as

$$
\begin{aligned}
& \text { C5: } \mathbf{S}_{\mathrm{C}_{5_{k}}}\left(\mathbf{W}, \mathbf{V}, \mathbf{W}_{\mathrm{E}}, \nu_{k}\right) \\
= & {\left[\begin{array}{cc}
\nu_{k} \mathbf{I}_{N_{\mathrm{T}}}+\mathbf{V} & \mathbf{V} \hat{\mathbf{g}}_{k} \\
\hat{\mathbf{g}}_{k}^{H} \mathbf{V} & -\nu_{k} \varepsilon_{k}^{2}-\frac{P_{\min }}{\eta}+\sigma_{\text {ant }}^{2}+\hat{\mathbf{g}}_{k}^{H} \mathbf{V} \hat{\mathbf{g}}_{k}
\end{array}\right] } \\
+\quad & \mathbf{U}_{\mathbf{g}_{k}}^{H} \mathbf{W}_{\mathrm{E}} \mathbf{U}_{\mathbf{g}_{k}}+\mathbf{U}_{\mathbf{g}_{k}}^{H} \mathbf{W} \mathbf{U}_{\mathbf{g}_{k}} \succeq \mathbf{0}, \forall k,
\end{aligned}
$$

for $\nu_{k} \geq 0, k \in\{1, \ldots, K-1\}$. We note that now constraints C2 and C5 involve only a finite number of constraints which facilitates the resource allocation algorithm design.

The third obstacle in solving (17) is the probabilistic (chance) constraint $\mathrm{C} 3$ and the problem formulation is known as chance constrained programming. In general, constraint $\mathrm{C} 3$ is non-convex since the optimization variables are coupled. To overcome this problem, we introduce the following lemma.
Lemma 2: Assuming the normalized upper bound channel gain vectors of the passive eavesdroppers, $\tilde{\mathbf{L}}_{j}, j \in\{1, \ldots, J\}$, can be modeled as independent and identical distributed (i.i.d.) Rayleigh random variables 10 , the following constraint implication holds:

$$
\begin{aligned}
& \overline{\mathrm{C} 3:}\left(\Phi_{N_{\mathrm{T}}}^{-1}\left(1-\kappa^{1 / J}\right) \Gamma_{\mathrm{tol}} \tilde{\sigma}^{2}\right) \mathbf{I}_{\mathrm{N}_{\mathrm{T}}} \\
& \succeq \mathbf{W}-\Gamma_{\mathrm{tol}} \mathbf{W}_{\mathrm{E}}-\Gamma_{\mathrm{tol}} \mathbf{V} \\
& \Longrightarrow \quad \mathrm{C} 3: \operatorname{Pr}\left(\max _{j \in\{1, \ldots, J\}}\left\{\Gamma_{\mathrm{PE}, j}\right\} \leq \Gamma_{\mathrm{tol}}\right) \geq \kappa,
\end{aligned}
$$

where $\Phi_{N_{\mathrm{T}}}^{-1}(\cdot)$ denotes the inverse cumulative distribution function (c.d.f.) of an inverse central chi-square random variable with $2 N_{\mathrm{T}}$ degrees of freedom.

Proof: Please refer to Appendix A for a proof of Lemma 2

As a result, we can replace 11 the chance constraint $\mathrm{C} 3$ with the constraint in 23 . Constraint $\overline{\mathrm{C} 3}$ is tractable in the sense that: (i) a feasible solution point satisfying (23) is also feasible for $\mathrm{C} 3$, and (ii) the new constraint in (23) is convex with respect to the optimization variables [28]. Thus, substituting (21) and (22) into (17) and replacing constraint C3 by (23), we obtain the following optimization problem:

$$
\begin{aligned}
& \underset{\mathbf{W}, \mathbf{V}, \mathbf{W}_{\mathrm{E}} \in \mathbb{H}^{N_{\mathrm{T}}, \rho, \boldsymbol{\delta}, \boldsymbol{\nu}}}{\operatorname{minimize}} \operatorname{Tr}(\mathbf{W})+\operatorname{Tr}(\mathbf{V})+\operatorname{Tr}\left(\mathbf{W}_{\mathrm{E}}\right) \\
& \text { s.t. } \mathrm{C} 1, \mathrm{C} 4, \mathrm{C} 6, \mathrm{C} 7, \mathrm{C} 8 \text {, } \\
& \mathrm{C} 2: \mathbf{S}_{\mathrm{C}_{2}}\left(\mathbf{W}, \mathbf{V}, \delta_{k}\right) \succeq \mathbf{0}, \forall k \text {, } \\
& \overline{\mathrm{C} 3}:\left(\Phi_{N_{\mathrm{T}}}^{-1}\left(1-\kappa^{1 / J}\right) \Gamma_{\mathrm{tol}} \tilde{\sigma}^{2}\right) \mathbf{I}_{\mathrm{N}_{\mathrm{T}}} \succeq \mathbf{W}-\Gamma_{\mathrm{tol}} \mathbf{W}_{\mathrm{E}}-\Gamma_{\mathrm{tol}} \mathbf{V} \text {, } \\
& \text { C5: } \mathbf{S}_{\mathrm{C}_{5_{k}}}\left(\mathbf{W}, \mathbf{V}, \mathbf{W}_{\mathrm{E}}, \nu_{k}\right) \succeq \mathbf{0}, \forall k \text {, } \\
& \text { C9: } \operatorname{Rank}(\mathbf{W})=1, \quad \text { C10: } \delta_{k}, \nu_{k} \geq 0, \forall k \text {, }
\end{aligned}
$$

where $\delta$ and $\boldsymbol{\nu}$ are auxiliary optimization variable vectors, whose elements $\delta_{k} \geq 0, k \in\{1, \ldots, K-1\}$, and $\nu_{k} \geq$ $0, k \in\{1, \ldots, K-1\}$, were introduced in (21) and (22), respectively. Now, C9: $\operatorname{Rank}(\mathbf{W})=1$ is the remaining obstacle in solving the problem in 25). By relaxing constraint C9: $\operatorname{Rank}(\mathbf{W})=1$, i.e., removing it from the problem formulation, the considered problem becomes a convex SDP which can be solved efficiently by numerical solvers such as SeDuMi [32] and SDPT3 [33]. From the basic principles of optimization theory, if the obtained solution $\mathbf{W}$ for the relaxed problem admits a rank-one matrix, then it is the optimal solution of the original problem in (25). Then, the optimal $\mathbf{w}$ can be obtained by performing eigenvalue decomposition on

\footnotetext{
${ }^{10} \mathrm{We}$ assume that the passive eavesdroppers are not collaborating and are physically separated by a distance of at least half a wavelength. For a carrier frequency of $470 \mathrm{MHz}$, half of a wavelength is roughly equal to $30 \mathrm{~cm}$ which is a reasonable assumption for the minimum receiver separation in practice. Besides, we assume that there is a sufficient number of scatterers in the channels between the transmitter and the passive eavesdroppers such that these channels can be modelled as Rayleigh distribution due to the central limit theorem [29]. These assumptions are commonly used in the literature [30], [31 when studying resource allocation algorithm design for providing physical layer security.

11 We note that a resource allocation policy which satisfies constraint $\overline{\mathrm{C} 3}$ also satisfies $\mathrm{C} 3$, but not vice versa. In other words, replacing constraint $\mathrm{C} 3$ with $\overline{\mathrm{C} 3}$ results in a smaller feasible solution set for optimization which leads to a lower bound system performance. To quantify the impact of the constraint restriction, the resulting performance loss is quantified in Section $\mathrm{V}$ via simulations.
} 
W. However, it is known that the constraint relaxation may not be tight and $\operatorname{Rank}(\mathbf{W})>1$ may occur. In the following, we will first reveal a sufficient condition for $\operatorname{Rank}(\mathbf{W})=1$ for the relaxed problem. Then, we propose a method for constructing an optimal solution for the relaxed version of 25 with a rank-one matrix W. Furthermore, we exploit the sufficient condition of the optimal solution and use it as a building block for the design of a suboptimal resource allocation algorithm, which requires a lower computational complexity than the construction of the rank-one solution.

\section{B. Optimality Conditions for SDP Relaxation}

In this subsection, we first reveal the tightness of the proposed SDP relaxation. Then, we study a sufficient condition for obtaining an optimal solution which will be used to design a low complexity suboptimal resource allocation scheme.

First, we introduce a theorem which reveals the tightness of the SDP relaxation. This theorem closely follows a similar theorem in [17, Proposition 4.1 12 .

Theorem 1: Suppose the optimal solution of the relaxed version of 25 is denoted as $\left\{\mathbf{W}^{*}, \mathbf{V}^{*}, \mathbf{W}_{\mathrm{E}}^{*}, \rho^{*}\right.$, $\left.\boldsymbol{\delta}^{*}, \boldsymbol{\nu}^{*}\right\}, \Gamma_{\text {req }}>0$, and $\operatorname{Rank}\left(\mathbf{W}^{*}\right)>1$. Then, there exists a feasible solution of (25), denoted as $\left\{\widetilde{\mathbf{W}}^{*}, \widetilde{\mathbf{V}}^{*}\right.$, $\left.\widetilde{\mathbf{W}}_{\mathrm{E}}^{*}, \widetilde{\rho}^{*}, \widetilde{\boldsymbol{\delta}}^{*}, \widetilde{\boldsymbol{\nu}}^{*}\right\}$, which not only achieves the same objective value as $\left\{\mathbf{W}^{*}, \mathbf{V}^{*}, \mathbf{W}_{\mathrm{E}}^{*}, \rho^{*}, \boldsymbol{\delta}^{*}, \boldsymbol{\nu}^{*}\right\}$, but also admits a rankone matrix $\widetilde{\mathbf{W}}^{*}$, i.e., $\operatorname{Rank}\left(\widetilde{\mathbf{W}}^{*}\right)=1$.

Proof: Please refer to Appendix B for a proof of Theorem 1 and the method for constructing $\left\{\widetilde{\mathbf{W}}^{*}, \widetilde{\mathbf{V}}^{*}, \widetilde{\mathbf{W}}_{\mathrm{E}}^{*}, \widetilde{\rho}^{*}, \widetilde{\boldsymbol{\delta}}^{*}, \widetilde{\boldsymbol{\nu}}^{*}\right\}$ with $\operatorname{Rank}\left(\widetilde{\mathbf{W}}^{*}\right)=1$.

As discussed in Appendix B, constructing an optimal solution set $\left\{\widetilde{\mathbf{W}}^{*}, \widetilde{\mathbf{V}}^{*}, \widetilde{\mathbf{W}}_{\mathrm{E}}^{*}, \widetilde{\rho}^{*}, \widetilde{\boldsymbol{\delta}}^{*}, \widetilde{\boldsymbol{\nu}}^{*}\right\}$ with $\operatorname{Rank}\left(\widetilde{\mathbf{W}}^{*}\right)=1$ may require the solution of the dual problem and one extra optimization problem, cf. Remark 6 Besides, knowledge of the Lagrange multiplier matrix $\mathbf{Y}^{*}$ is required for constructing the rank-one solution which may not be provided by some numerical solvers. Furthermore, the associated signal processing delay and computational complexity may not be affordable when the transmitter has limited signal processing capability. Thus, in the following, we present a sufficient condition for obtaining a rank-one matrix solution $\mathbf{W}$ for the relaxed version of problem 25 which will also be used for designing a low complexity suboptimal resource allocation algorithm.

Proposition 1: Consider the relaxed version of problem (25) for $\Gamma_{\text {req }}>0$ and denote $\mathbf{D}_{\mathrm{C}_{2_{k}}}^{*}$ and $\mathbf{D}_{\mathrm{C}_{5_{k}}}^{*}$ as the optimal Lagrange multiplier matrices associated with constraints $\mathrm{C} 2$ and C5, respectively. A sufficient condition for $\operatorname{Rank}\left(\mathbf{W}^{*}\right)=$ 1 is that $\frac{\mathbf{D}_{\mathrm{C}_{2}}^{*}}{\Gamma_{\mathrm{tol}_{k}}}-\mathbf{D}_{\mathrm{C}_{5_{k}}}^{*} \succeq \mathbf{0}, \forall k \in\{1, \ldots, K-1\}$, holds. 1

Proof: Please refer to Appendix C for a proof of Proposition

Intuitively, when the requirement in constraint $\mathrm{C} 5$ becomes less stringent, i.e., $P_{\min _{k}} \rightarrow 0$, the corresponding Lagrange multiplier matrix $\mathbf{D}_{\mathrm{C}_{5_{k}}}^{*} \rightarrow \mathbf{0}$ since the constraint is less

\footnotetext{
${ }^{12}$ We note that the theorem in [17. Proposition 4.1] considers a communication system with separated receivers for the case of perfect CSI and without passive eavesdroppers. In fact, the results of [17 cannot be directly applied to the system model considered in this paper without the steps and transformations introduced in Sections II to IV-A.
}

often active. As a result, the SDP relaxation algorithm has a higher chance of obtaining a rank-one matrix solution and thus achieves the global optimum with respect to the reformulated problem.

Remark 5: We would like to emphasize that although Proposition 1 provides a sufficient condition for a rank-one matrix solution under SDP relaxation, we found by simulation that the proposed SDP relaxation may result in a rank-one matrix $\mathbf{W}^{*}$ even if the sufficient condition does not hold.

Suboptimal resource allocation scheme: According to Proposition 1, the solution of (25) has a rank-one $\mathbf{W}^{*}$ when constraint $\mathrm{C} 5$ is not active, i.e., $\mathbf{D}_{\mathrm{C}_{5 k}}^{*}=\mathbf{0}, \forall k$, or constraint $\mathrm{C} 5$ is independent of optimization variable $\mathbf{W}$. For facilitating an efficient resource allocation algorithm design, we replace constraint $\mathrm{C} 5$ in $(25)$ by $\overline{\mathrm{C} 5}$ and the new optimization problem becomes

$$
\begin{aligned}
& \underset{\mathbf{V}, \mathbf{W}_{\mathrm{E}} \in \mathbb{H}^{N_{\mathrm{T}}, \rho, \boldsymbol{\delta}, \boldsymbol{\nu}}}{\operatorname{minimize}} \operatorname{Tr}(\mathbf{W})+\operatorname{Tr}(\mathbf{V})+\operatorname{Tr}\left(\mathbf{W}_{\mathrm{E}}\right) \\
& \text { s.t. } \quad \mathrm{C} 1, \mathrm{C} 2, \overline{\mathrm{C}} 3, \mathrm{C} 4-\mathrm{C} 8, \mathrm{C} 10 \text {, } \\
& \overline{\mathrm{C} 5}: \tilde{\mathbf{S}}_{\mathrm{C}_{5_{k}}}\left(\mathbf{V}, \mathbf{W}_{\mathrm{E}}, \nu_{k}\right) \\
& =\left[\begin{array}{cc}
\nu_{k} \mathbf{I}_{N_{\mathrm{T}}}+\mathbf{V} & \mathbf{V} \hat{\mathbf{g}}_{k} \\
\hat{\mathbf{g}}_{k}^{H} \mathbf{V} & -\nu_{k} \varepsilon_{k}^{2}-\frac{P_{\min }}{\eta}+\sigma_{\mathrm{ant}}^{2}+\hat{\mathbf{g}}_{k}^{H} \mathbf{V} \hat{\mathbf{g}}_{k}
\end{array}\right] \\
& +\quad \mathbf{U}_{\mathbf{g}_{k}}^{H} \mathbf{W}_{\mathrm{E}} \mathbf{U}_{\mathbf{g}_{k}} \succeq \mathbf{0}, \forall k \text {. }
\end{aligned}
$$

Compared to constraint $\mathrm{C} 5$, the new constraint $\overline{\mathrm{C} 5}$ reduces the feasible solution set for 25 as the contribution of the information signal to the energy harvesting, i.e., $\mathbf{W}$, at idle receiver $k$ is neglected. Thus, the obtained solution of problem (26) serves as a performance lower bound for the reformulated optimization problem 25]. We note that the new constraint preserves the convexity of the relaxed problem and (26) can be solved efficiently via SDP relaxation and the aforementioned numerical solvers. Furthermore, it can be shown that the obtained solution 13 of problem (26) has always a rank-one beamforming matrix, i.e., $\operatorname{Rank}(\mathbf{W})=1$, even though SDP relaxation is applied.

\section{RESUlts}

In this section, we evaluate the system performance for the proposed resource allocation schemes using simulations. The TGn path loss model [34] for indoor communications is adopted with a carrier center frequency of $470 \mathrm{MHz}$ [35]. The directional joint transmit and receive antenna gain is 10 $\mathrm{dB}$. The reference distance of the path loss model is 2 meters and there are $K$ receivers uniformly distributed between the reference distance and the maximum service distance of 20 meters. The wavelength of the carrier signal is 0.6 meter which is smaller than the minimum distance between the transmitter and the receivers. Thus, the far-field assumption for the channel model in [34] holds. The transmitter is equipped with $N_{\mathrm{T}}$ antennas. The small scale fading coefficients between

\footnotetext{
${ }^{13}$ We can follow a similar approach as in Appendix $\mathrm{C}$ to examine the KKT conditions for the new problem formulation in 26. In particular, the sufficient condition for a rank-one matrix solution stated in Proposition 1 is always satisfied for the new problem formulation since constraint $\overline{\mathrm{C} 5}$ is independent of $\mathbf{W}$.
} 


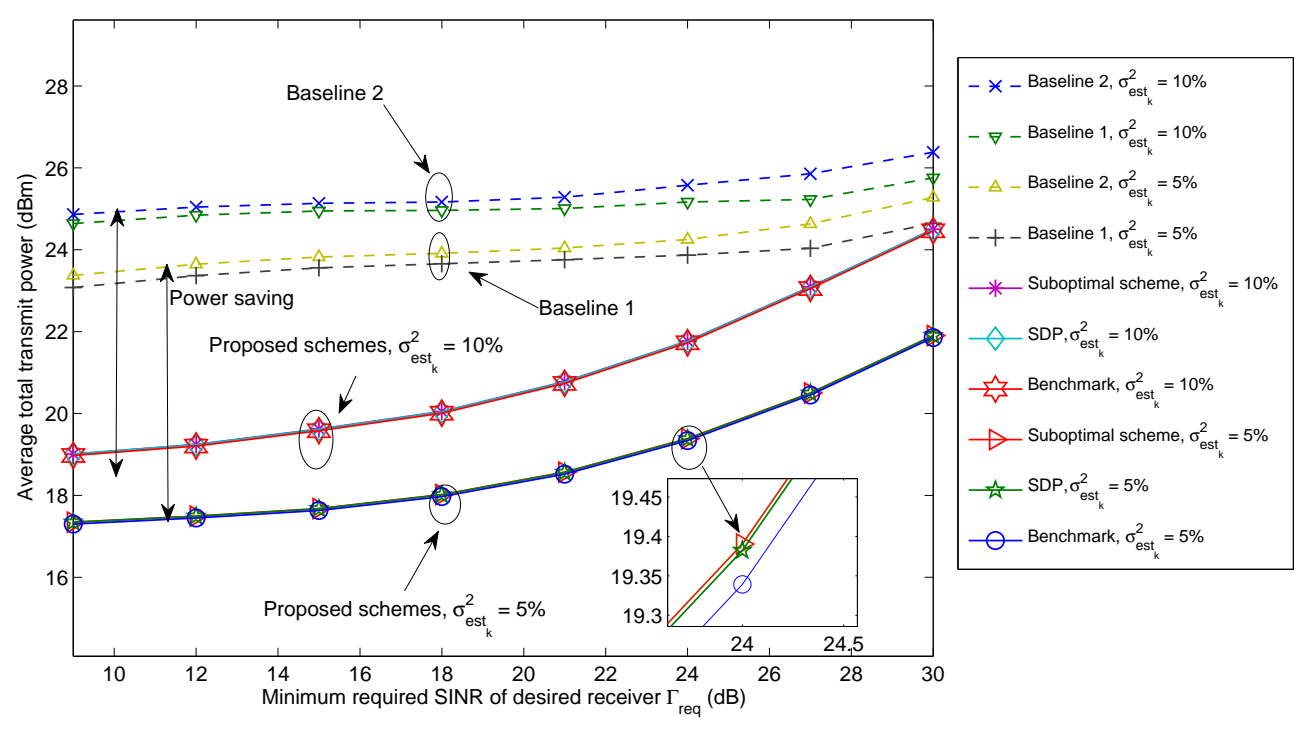

Fig. 2. Average total transmit power $(\mathrm{dBm})$ versus the minimum required SINR of the desired receiver, $\Gamma_{\text {req }}(\mathrm{dB})$, for different resource allocation schemes. The double-sided arrows indicate the power savings achieved by the proposed schemes compared to the baseline schemes.

the transmitter and the legitimate receivers are generated as independent and identically distributed Rician random variables with Rician factor $6 \mathrm{~dB}$. The corresponding shadowing effect is assumed to be $0 \mathrm{~dB}$. On the other hand, we assume that there are $J=5$ eavesdroppers eavesdropping the information from outdoors. Thus, the multipath fading coefficients between the transmitter and the $J$ passive eavesdroppers are modeled as Rayleigh random variables. We set $\kappa=0.99$ for providing secure communication. We assume that the signal processing noise powe 14 and the antenna noise power are $\sigma_{\mathrm{s}}^{2}=-35$ $\mathrm{dBm}$ and $\sigma_{\text {ant }}^{2}=-111 \mathrm{dBm}$, respectively. Unless specified otherwise, we assume a minimum required power transfer of $P_{\min }=P_{\min _{k}}=0 \mathrm{dBm}, \forall k$, and an energy harvesting efficiency of $\eta=0.5$. On the other hand, the maximum SINR tolerance of each idle receiver (potential eavesdropper) and passive eavesdropper is set to $\Gamma_{\mathrm{tol}_{k}}=\Gamma_{\text {tol }}=0 \mathrm{~dB}$. The maximum transmit power per-antenna is set to $P_{\max _{n}}=30$ $\mathrm{dBm}, \forall n \in\left\{1, \ldots, N_{\mathrm{T}}\right\}$. To facilitate the presentation in the sequel, we define the normalized maximum channel estimation error of idle receiver $k$ as $\sigma_{\text {est }_{k}}^{2}=\frac{\varepsilon_{k}^{2}}{\left\|g_{k}\right\|^{2}}$ where $\sigma_{\text {est }_{a}}^{2}=\sigma_{\text {est }_{b}}^{2}, \forall a, b \in\{1, \ldots, K-1\}$. The average system performance shown in the following sections is obtained by averaging over different realizations of both path loss and multipath fading.

\footnotetext{
${ }^{14} \mathrm{We}$ assume that the signal processing noise power at each receiver is due to quantization noise in the analog-to-digital (ADC) converter. Specifically, a 10-bit uniform quantizer is used for quantizing the received signal at the receivers.
}

\section{A. Average Total Transmit Power versus Minimum Required SINR}

Figure 2 depicts the average total transmit power versus the minimum required SINR of the desired receiver, $\Gamma_{\text {req }}$, for $K=$ 4 legitimate receivers, different resource allocation schemes, and different normalized maximum channel estimation errors, $\sigma_{\text {est }_{k}}^{2}$. The transmitter has $N_{\mathrm{T}}=6$ transmit antennas. It can be observed that the average total transmit power of the proposed SDP resource allocation scheme is a monotonically nondecreasing function of $\Gamma_{\text {req. }}$. This is attributed to the fact that a higher transmit power is necessary for satisfying constraint $\mathrm{C} 1$ when the minimum SINR requirement of $\Gamma_{\text {req }}$ becomes more stringent. Besides, it can be seen that the average total transmit power increases for increasing normalized maximum channel estimation errors, $\sigma_{\text {est }_{k}}^{2}$. In fact, with increasing imperfectness of the channel estimation, the transmitter has to allocate more power to the artificial noise and the energy signal to prevent interception by potential eavesdroppers and to facilitate efficient energy transfer for fulling constraints $\mathrm{C} 2$ and $\mathrm{C} 5$, respectively. Furthermore, we investigate the performance loss incurred by replacing the non-convex probabilistic constraint $\mathrm{C} 3$ with the proposed convex deterministic constraint $\overline{\mathrm{C} 3}$, cf. Lemma 2 and 25), in Figure 2 In particular, we compare the performance of the proposed optimal SDP resource allocation scheme obtained based on 25) with that of a benchmark system. Specifically, the performance of the benchmark system is computed by solving (16) for $\kappa=0$ in constraint C3. In other words, we assume that the passive eavesdropper does not exist in the system. Thus, the performance gap between the curves of the SDP resource allocation scheme and the benchmark system constitutes an upper bound on the performance loss incurred 
by the constraint replacement. Figure 2 shows that for a wide range of minimum required SINRs of the desired receiver, the performance loss is less than $0.1 \mathrm{~dB}$, despite the fact that the reformulated problem guarantees communication security robustness against passive eavesdropping.

For comparison, Figure 2] also contains the average total transmit power of the proposed suboptimal scheme and two baseline resource allocation schemes. For baseline scheme 1, we adopt an isotropic radiation pattern for $\mathbf{W}_{\mathrm{E}}$ as the CSI of the passive eavesdroppers is not known at the transmitter [13]. Then, we minimize the total transmitted power by optimizing $\mathbf{W}, \mathbf{V}, \rho, \boldsymbol{\delta}, \boldsymbol{\nu}$, and the power allocated to $\mathbf{w}_{\mathrm{E}}$ subject to the same constraints as in (25) via SDP relaxation. For baseline scheme 2, we adopt maximum ratio transmission (MRT) [2] for delivering the information signal to the desired legitimate receiver. Similar to baseline scheme 1, an isotropic radiation pattern is adopted for the energy signal. Then, we optimize the power allocated to $\mathbf{W}_{\mathrm{E}}$, the MRT beamforming matrix $\mathbf{W}, \mathbf{V}, \rho, \boldsymbol{\delta}$, and $\boldsymbol{\nu}$ for minimization of the total transmit power subject to the same constraints as in 25). It can be seen that the proposed suboptimal scheme closely approaches the performance of the benchmark system and the performance achieved by SDP relaxation of the reformulated problem, despite the fact that the proposed suboptimal scheme employs a smaller feasible solution set compared to the original problem formulation in (16). On the other hand, it can be observed that the lower computational complexity of the baseline schemes comes at the expense of a significantly higher transmit power compared to the other schemes. Indeed, the proposed SDP resource allocation scheme and the suboptimal scheme fully utilize the CSI of all communication links and optimize the space spanned by the energy signal and the artificial noise for performing resource allocation. On the contrary, for the baseline schemes, the transmitter is unable to fully exploit the available degrees of freedom in resource allocation since the energy signal is radiated isotropically and/or $\mathbf{W}$ is fixed. Yet, even though the available CSI at the transmitter is imperfect, all the schemes (including the baseline schemes) are able to fulfill the QoS requirements due to the proposed robust resource allocation algorithm design and the optimization of the artificial noise covariance matrix $\mathbf{V}$.

Figure 3(a) and Figure 3(b) illustrate the average system secrecy capacity versus the minimum required SINR of the desired receiver, $\Gamma_{\text {req }}$, for $K=4$ receivers, $N_{\mathrm{T}}=6$ transmit antennas, different resource allocation schemes, and different normalized maximum channel estimation errors, $\sigma_{\mathrm{est}_{k}}^{2}$. It can be seen that the average system secrecy capacity, i.e., $C_{\mathrm{sec}}$, increases with $\Gamma_{\text {req }}$ since the maximum tolerable SINRs of the idle receivers are constrained to $\Gamma_{\text {tol }_{k}}=0 \mathrm{~dB}$. Besides, as expected, the baseline schemes achieve on average a higher secrecy capacity in the high transmit power regime compared to the other schemes. However, the superior performance in terms of secrecy capacity comes at the expense of an exceedingly high transmit power, cf. Figure 2 since the transmitter is forced to transmit more artificial noise and a stronger energy signal for delivering energy to the idle receivers. On the other hand, the normalized maximum channel estimation errors $\sigma_{\text {est }_{k}}^{2}$ do not have a large impact on the average secrecy

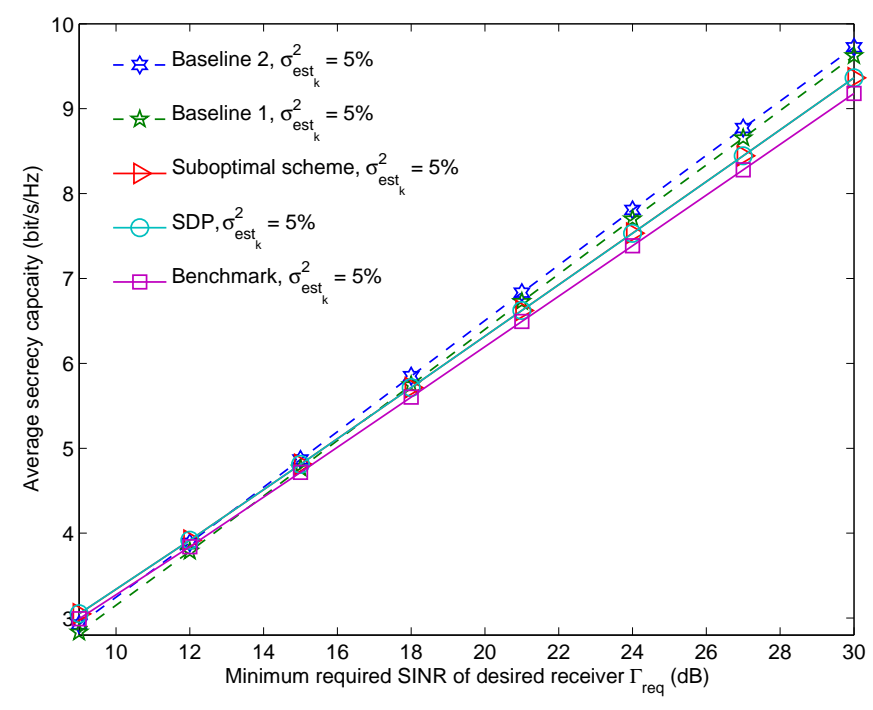

(a) Normalized maximum channel estimation errors $\sigma_{\text {est }_{k}}^{2}=5 \%$.

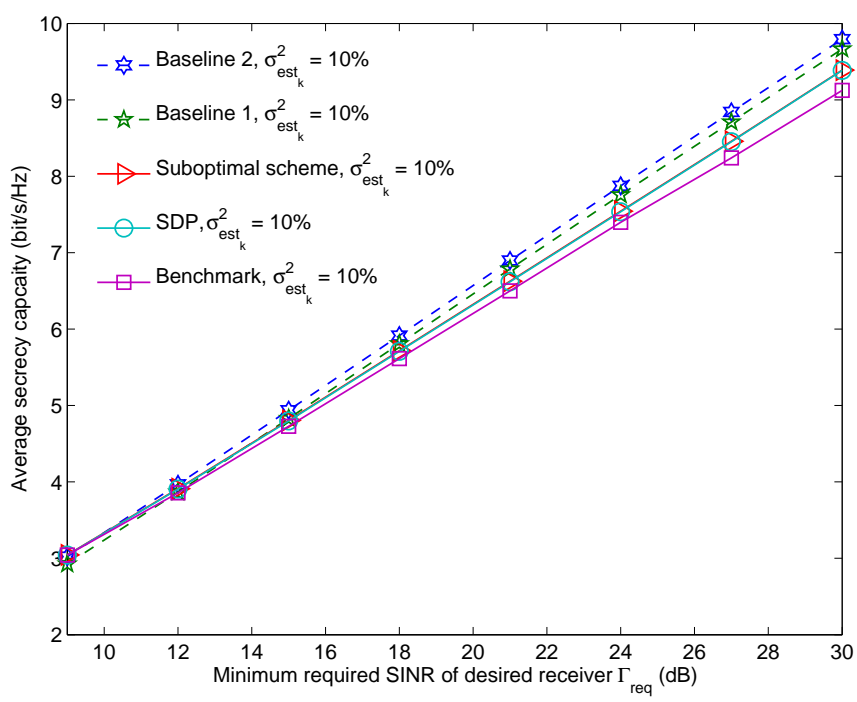

(b) Normalized maximum channel estimation errors $\sigma_{\text {est }_{k}}^{2}=10 \%$.

Fig. 3. Average system secrecy capacity (bit/s/Hz) versus the minimum required SINR of the desired receiver, $\Gamma_{\text {req }}(\mathrm{dB})$, for different normalized maximum channel estimation errors $\sigma_{\text {est }_{k}}^{2}$, and different resource allocation schemes.

capacity of the system and all schemes are able to guarantee the secrecy QoS because of the proposed robust optimization.

\section{B. Average Total Transmit Power versus Number of Transmit Antennas}

Figure 4(a) shows the average total transmit power versus the number of transmit antennas, $N_{\mathrm{T}}$, for $K=4$ legitimate receivers, different resource allocation schemes, and a normalized maximum channel estimation error of $\sigma_{\text {est }_{k}}^{2}=5 \%, \forall k$. The minimum required SINR of the desired receiver is set to $\Gamma_{\text {req }}=15 \mathrm{~dB}$. It is expected that the total transmit power decreases with an increasing number of antennas since extra degrees of freedom can be exploited for resource allocation 
when more antennas are available at the transmitter. On the other hand, the proposed schemes provide substantial power savings compared to the two baseline schemes due to the optimization of both $\mathbf{W}$ and $\mathbf{W}_{\mathrm{E}}$. Figure 4(b) depicts the average transmit power allocation to the three components of the transmitted signal, i.e., $\operatorname{Tr}(\mathbf{W}), \operatorname{Tr}(\mathbf{V})$, and $\operatorname{Tr}\left(\mathbf{W}_{\mathrm{E}}\right)$, versus the number of transmit antennas for the proposed SDP resource allocation scheme and baseline scheme 2 under the same system setting as considered in Figure 4(a). It can be observed that the amounts of power allocated to the information signal and the artificial noise decrease rapidly for the SDP resource allocation scheme as the number of transmit antennas increases. In fact, the degrees of freedom for resource allocation increase with the number of transmit antennas. Specifically, with more antennas, the transmitter is able to perform more power efficient beamforming of the information signal and more effective jamming of the potential and passive eavesdroppers. Besides, the power allocated to the energy signal decreases with increasing $N_{\mathrm{T}}$ but not as fast as the artificial noise power. This is because the energy signal has less influence on the system performance than the artificial noise. In particular, the energy signal does not degrade the received SINRs at the potential eavesdroppers. On the other hand, the powers allocated to the information signal and the artificial noise do not vary significantly with $N_{\mathrm{T}}$ in baseline scheme 2 since the transmitter is unable to fully exploit the extra degrees of freedom introduced by additional transmit antennas.

\section{Average Total Transmit Power versus Number of Legitimate Receivers}

Figure 5(a) shows the average total transmit power versus the number of legitimate receivers, $K$, for a normalized maximum channel estimation error of $\sigma_{\mathrm{est}_{k}}^{2}=5 \%, \forall k$, and different resource allocation schemes. The minimum required SINR of the desired receiver is set to $\Gamma_{\text {req }}=15 \mathrm{~dB}$ and there are $N_{\mathrm{T}}=6$ transmit antennas. It is expected that the total transmit power increases with the number of legitimate receivers. The reason behind this is twofold. First, as the number of legitimate receivers in the system increases, there are more idle receivers requiring power transfer from the transmitter even though some of them are experiencing bad channel conditions. Second, there are more potential eavesdroppers present in the system and thus the transmitter has to generate a higher amount of artificial noise to guarantee communication secrecy. On the other hand, the proposed schemes provide substantial power savings compared to the two baseline schemes. Figure 5(b) shows the average transmit power allocated the different components of the transmitted signal. We focus on the power allocation for the proposed SDP resource allocation scheme and baseline scheme 2 . It can be observed that a large portion of power is allocated to the energy signal and artificial noise in both schemes. This is due to the fact that when there are more potential eavesdroppers (idle legitimate receivers) in the system, the secrecy QoS and energy harvesting requirements dominate the system performance as constraints $\mathrm{C} 2$ and C5 become more stringent. This translates to a higher demand

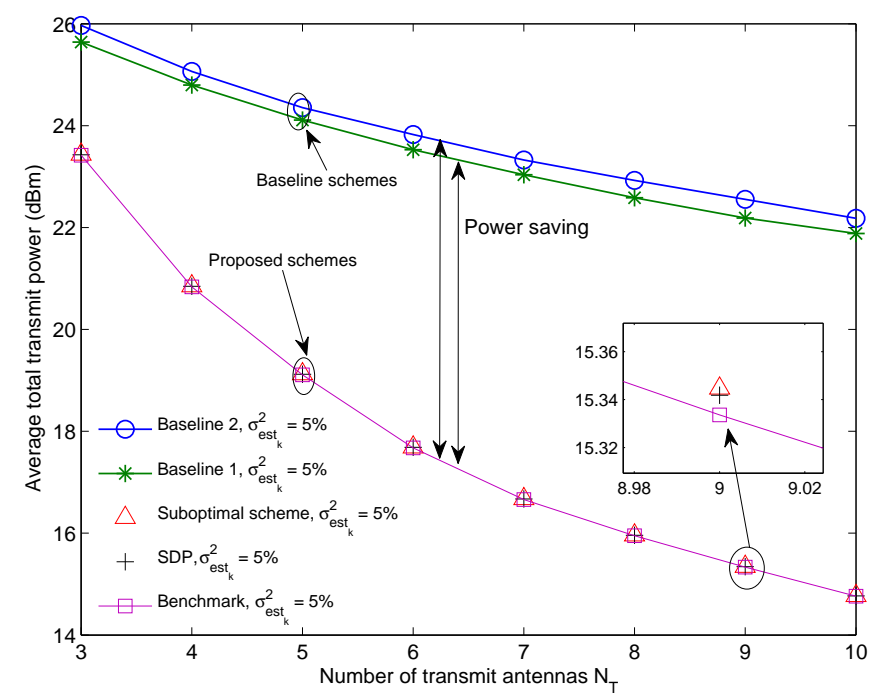

(a) Average total transmit power.

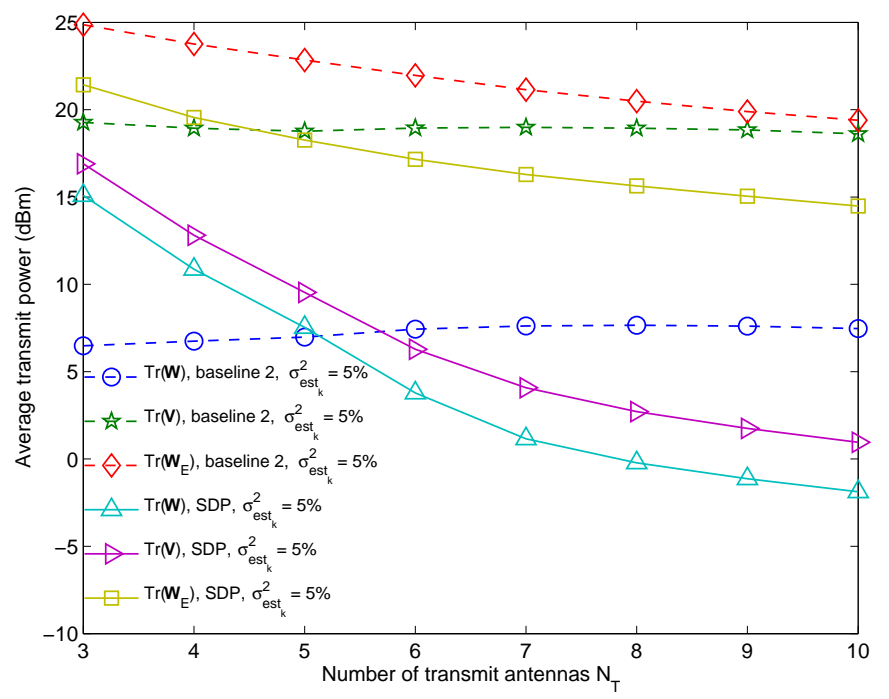

(b) Average transmit power allocation.

Fig. 4. Average total transmit power $(\mathrm{dBm})$ and average transmit power allocation $(\mathrm{dBm})$ versus the number of transmit antennas, $N_{\mathrm{T}}$, for $\Gamma_{\mathrm{req}}=15$ $\mathrm{dB}$, a normalized maximum channel estimation error of $\sigma_{\mathrm{est}_{k}}^{2}=5 \%$, and different resource allocation schemes. The double-sided arrows indicate the power savings achieved by the proposed schemes compared to the baseline schemes.

for artificial noise and energy signal generation (/radiation) for degrading the channels of the potential eavesdroppers and facilitating efficient power transfer. Besides, it is interesting to note that the amount of power allocated by baseline scheme 2 to the information signal decreases with increasing number of legitimate receivers. This result suggests that with MRT allocating a larger amount of power to the information signal is not effective in minimizing the total transmit power. Instead, a larger portion of the transmit power should be allocated to the artificial noise and the energy signal for jamming the channel of the potential eavesdroppers when there are more legitimate receivers in the system. 


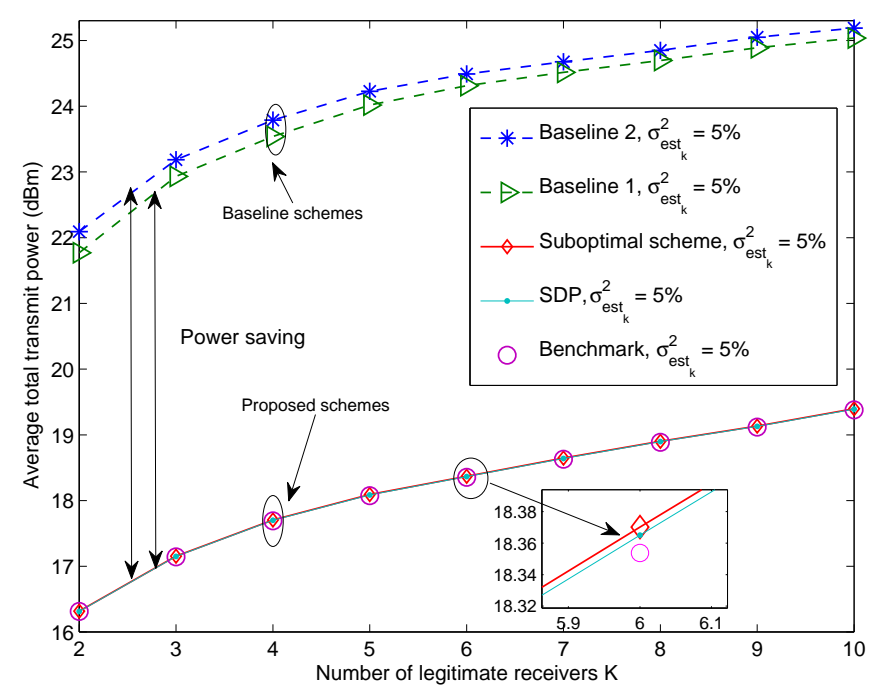

(a) Average total transmit power.

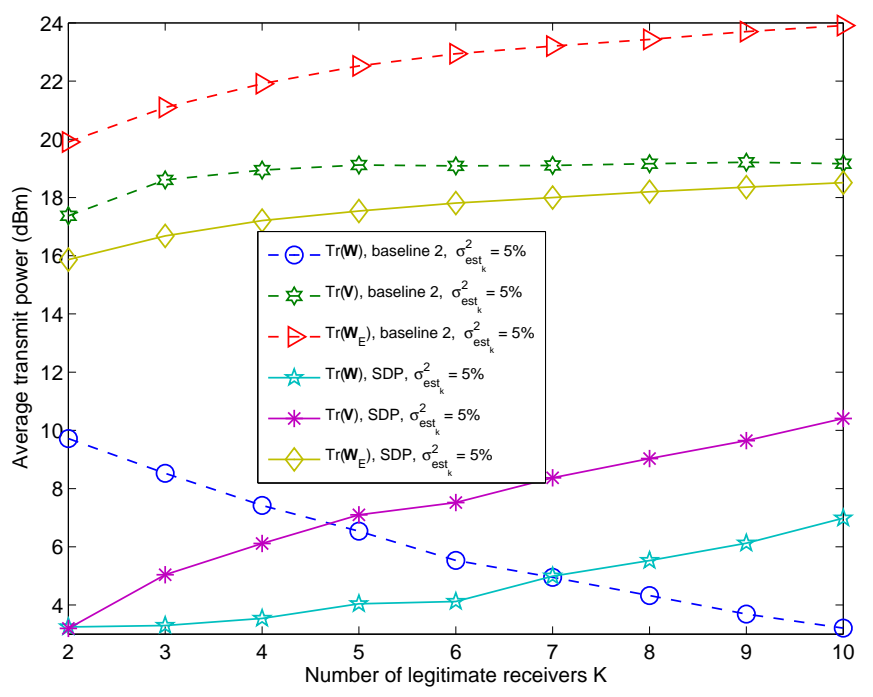

(b) Average transmit power allocation.

Fig. 5. Average total transmit power $(\mathrm{dBm})$ versus the number of legitimate receivers, $K$, for $\Gamma_{\text {req }}=15 \mathrm{~dB}$, a normalized maximum channel estimation error of $\sigma_{\text {est }_{k}}^{2}=5 \%$, and different resource allocation schemes. The doublesided arrows indicate the power savings achieved by the proposed schemes compared to the baseline schemes.

\section{Average Total Harvested Power}

Figure 6(a) shows the average total harvested power versus the minimum required SINR of the desired receiver, $\Gamma_{\text {req }}$, for $K=4$ legitimate receivers, $N_{\mathrm{T}}=6$ transmit antennas, different normalized maximum channel estimation errors, $\sigma_{\text {est }_{k}}^{2}$, and different resource allocation schemes. The average total harvested power is computed by assuming the potential eavesdroppers do not eavesdrop. It can be observed that the total average harvested power increases with $\Gamma_{\text {req. }}$. On the one hand, the transmitter has to allocate more power to the information bearing signal to satisfy a more stringent requirement on $\Gamma_{\text {req. }}$. On the other hand, the power of the artificial noise and energy

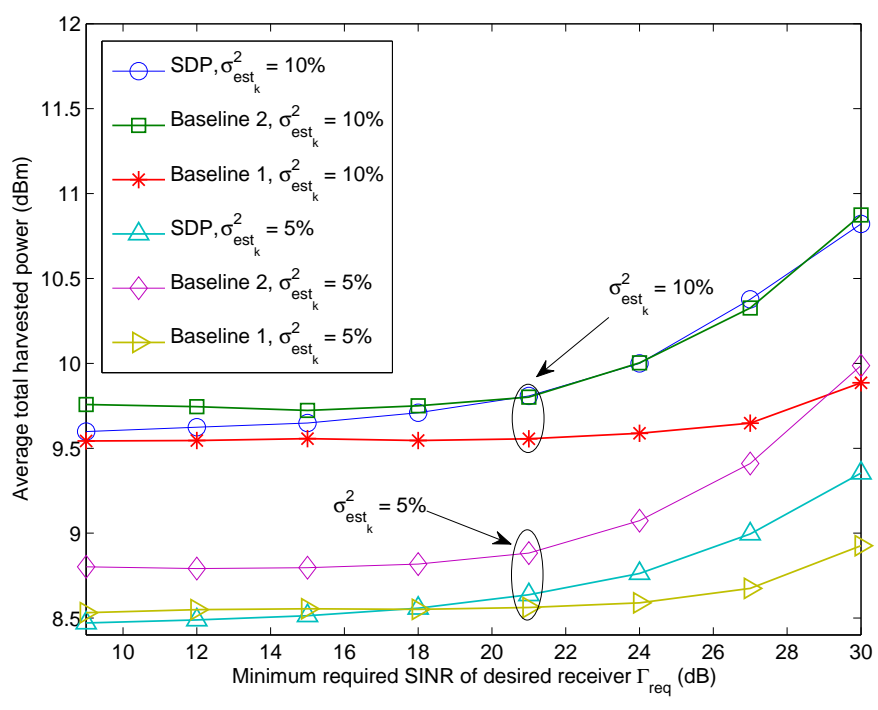

(a) Average total harvested power versus $\Gamma_{\text {req. }}$.

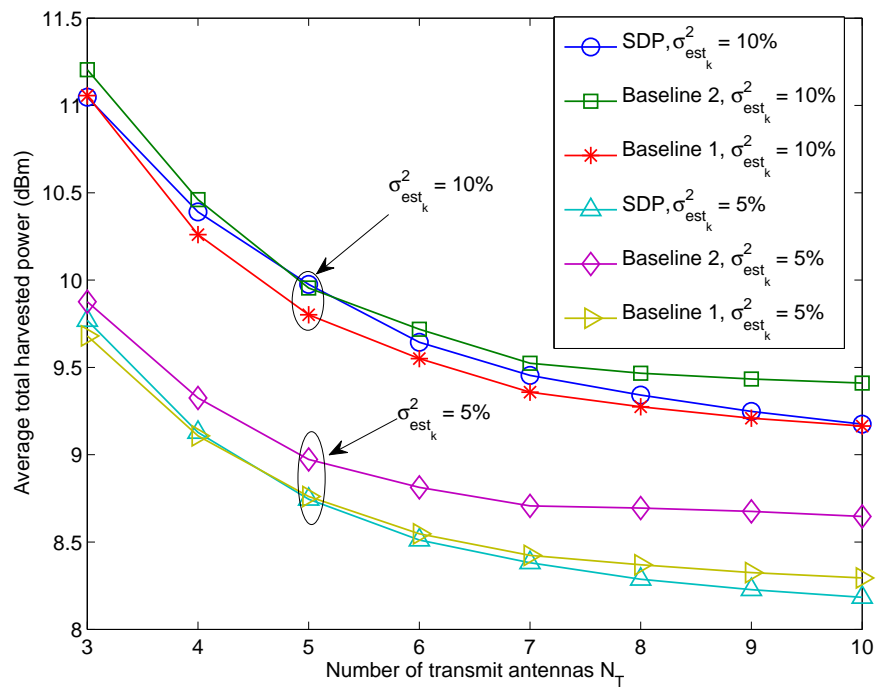

(b) Average total harvested power versus $N_{\mathrm{T}}$.

Fig. 6. Average total harvested power $(\mathrm{dBm})$ versus the minimum required SINR of the desired receiver, $\Gamma_{\text {req }}(\mathrm{dB})$, and the number of transmit antennas, $N_{\mathrm{T}}$, respectively, for $K=4$ legitimate receivers.

signal may also increase to reduce the received SINRs at both the potential eavesdroppers and the passive eavesdroppers. As a result, for larger $\Gamma_{\text {req }}$, more power is available in the RF and can be harvested by the legitimate receivers. Besides, it can be observed that the average total harvested power increases with increasing $\sigma_{\text {est }_{k}}^{2}$. In fact, to fulfill the QoS requirements on the minimum power transfer and communication secrecy, a higher amount of transmit power is required for larger $\sigma_{\text {est }_{k}}^{2}$ which leads to a higher energy level in the RF for energy harvesting. Figure 6(b) illustrates the average total harvested power versus the number of transmit antennas, $N_{\mathrm{T}}$, for $K=4$ legitimate receivers, $\Gamma_{\text {req }}=15 \mathrm{~dB}$, different normalized maximum channel estimation errors, $\sigma_{\text {est }_{k}}^{2}$, and 
different resource allocation schemes. It can be observed that the average total harvested power in the system decreases with increasing number of antennas for all considered scenarios. These results suggest that a lower amount of energy is available in the RF for energy harvesting when the number of transmit antennas increases. This is due to the fact that with more transmit antennas the direction of beamforming matrix $\mathbf{W}$ can be more accurately steered towards the desired receiver which reduces the power leakage of $\mathbf{W}$ to the idle receivers for energy harvesting. Besides, by exploiting the extra degrees of freedoms introduced by additional transmit antennas, the artificial noise and energy signal powers radiated from the transmitter to fulfill the secrecy QoS requirements can be reduced which further decreases the amount of energy available in the RF for energy harvesting. Yet, because of the proposed optimization, the proposed schemes are able to guarantee the minimum required power transfer to the legitimate receivers for all the cases studied.

\section{CONCLUSIONS}

In this paper, we formulated the resource allocation algorithm design for secure MISO communication systems with $\mathrm{RF}$ energy harvesting receivers as a non-convex optimization problem. The proposed problem formulation advocates the dual use of artificial noise and energy signals for facilitating simultaneous secure communication and efficient energy transfer in the presence of both passive eavesdroppers and potential eavesdroppers. Due to the intractability of the resulting power minimization problem, the problem was reformulated by replacing a non-convex probabilistic constraint with a convex deterministic constraint. Subsequently, an efficient SDP based resource allocation algorithm was proposed to obtain the global optimal solution for the reformulated problem. Besides a suboptimal low computational complexity resource allocation scheme was provided. Simulation results showed the excellent performance of all the proposed resource allocation schemes. Furthermore, our results also unveiled the power savings enabled by the optimization of the artificial noise and energy signal generation. An interesting topic for future work is the consideration of multiple antennas eavesdroppers and multiple desired receivers.

\section{APPENDIX}

\section{A. Proof of Lemma 2}

Since the channels of the $J$ passive eavesdroppers are independent, the left hand side of constraint C3 in (17) can be written as

$$
\begin{aligned}
& \operatorname{Pr}\left(\max _{j \in\{1, \ldots, J\}}\left\{\Gamma_{\mathrm{PE}, j}\right\} \leq \Gamma_{\text {tol }}\right) \\
= & \prod_{j=1}^{J} \operatorname{Pr}\left(\Gamma_{\mathrm{PE}, j} \leq \Gamma_{\text {tol }}\right) .
\end{aligned}
$$

Thus, after some mathematical manipulations, constraint C3 is equivalent to

$$
\begin{gathered}
\text { C3: } \operatorname{Pr}\left(\max _{j \in\{1, \ldots, J\}}\left\{\Gamma_{\mathrm{PE}, j}\right\} \leq \Gamma_{\mathrm{tol}}\right) \geq \kappa \\
\Leftrightarrow \operatorname{Pr}\left(\Gamma_{\mathrm{tol}} \tilde{\sigma}^{2} \geq \operatorname{Tr}\left(\tilde{\mathbf{L}}\left(\mathbf{W}-\Gamma_{\mathrm{tol}} \mathbf{W}_{\mathrm{E}}-\Gamma_{\mathrm{tol}} \mathbf{V}\right)\right)\right) \geq \kappa^{1 / J} .
\end{gathered}
$$

Since the equivalent channels of the passive eavesdroppers are modeled as i.i.d. random variables, we have dropped the index of the passive eavesdropper channels in 28) without loss of generality. Now, we focus on the calculation of the probability $\operatorname{Pr}\left(\Gamma_{\text {tol }} \tilde{\sigma}^{2} \geq \operatorname{Tr}\left(\tilde{\mathbf{L}}\left(\mathbf{W}-\Gamma_{\text {tol }} \mathbf{W}_{\mathrm{E}}-\Gamma_{\text {tol }} \mathbf{V}\right)\right)\right)$. It can be shown that the distribution of random variable $\operatorname{Tr}(\tilde{\mathbf{L}}(\mathbf{W}-$ $\left.\left.\Gamma_{\mathrm{tol}} \mathbf{W}_{\mathrm{E}}-\Gamma_{\mathrm{tol}} \mathbf{V}\right)\right)$ is the same as that of random variable

$$
\sum_{n=1}^{N_{\mathrm{T}}} \chi_{n}^{2} \lambda_{n}\left(\mathbf{W}-\Gamma_{\mathrm{tol}} \mathbf{W}_{\mathrm{E}}-\Gamma_{\mathrm{tol}} \mathbf{V}\right)
$$

where $\chi_{n}^{2}, n \in\left\{1, \ldots, N_{\mathrm{T}}\right\}$, are i.i.d. exponential random variables with probability density function $f_{\chi_{n}^{2}}(x)=\exp (-x)$ for $x \geq 0$. It can be seen in 29) that the $\chi_{n}^{2}$ are coupled with the optimization variable matrices and the distribution of the random variable in 29 depends on the value of the optimization variables $\left\{\mathbf{W}, \mathbf{W}_{\mathrm{E}}, \mathbf{V}\right\}$. On the other hand, the calculation of the optimization variables depends on the distribution of random variable in 29. This mutual dependence does not facilitate an efficient resource allocation algorithm design. Besides, matrix $\mathbf{W}-\Gamma_{\text {tol }} \mathbf{W}_{\mathrm{E}}-\Gamma_{\text {tol }} \mathbf{V}$ is in general indefinite and the resulting probability distribution of $\operatorname{Tr}\left(\tilde{\mathbf{L}}\left(\mathbf{W}-\Gamma_{\text {tol }} \mathbf{W}_{\mathrm{E}}-\Gamma_{\text {tol }} \mathbf{V}\right)\right)$ may not be a convex function. As a comprise solution, we focus on a smaller convex feasible solution set which facilitates a tractable solution. We first introduce a trace inequality for the product of two arbitrary Hermitian matrices [36]. In particular, for any two Hermitian matrices $\mathbf{A} \in \mathbb{H}^{N}$ and $\mathbf{B} \in \mathbb{H}^{N}$, the following inequality holds:

$$
\sum_{n=1}^{N} \lambda_{n}(\mathbf{A}) \lambda_{N-n+1}(\mathbf{B}) \leq \operatorname{Tr}(\mathbf{A B}) \leq \sum_{n=1}^{N} \lambda_{n}(\mathbf{A}) \lambda_{n}(\mathbf{B}) .
$$

Then, an upper bound for function $\operatorname{Tr}\left(\tilde{\mathbf{L}}\left(\mathbf{W}-\Gamma_{\text {tol }} \mathbf{W}_{\mathrm{E}}-\right.\right.$ $\left.\Gamma_{\text {tol }} \mathbf{V}\right)$ ) is given by

$$
\begin{array}{ll} 
& \operatorname{Tr}\left(\tilde{\mathbf{L}}\left(\mathbf{W}-\Gamma_{\text {tol }} \mathbf{W}_{\mathrm{E}}-\Gamma_{\text {tol }} \mathbf{V}\right)\right) \\
\stackrel{(a)}{\leq} & \sum_{n=1}^{N_{\mathrm{T}}} \lambda_{n}(\tilde{\mathbf{L}}) \lambda_{n}\left(\mathbf{W}-\Gamma_{\text {tol }} \mathbf{W}_{\mathrm{E}}-\Gamma_{\text {tol }} \mathbf{V}\right) \\
\stackrel{(b)}{=} & \lambda_{\max }(\tilde{\mathbf{L}}) \lambda_{\max }\left(\mathbf{W}-\Gamma_{\mathrm{tol}} \mathbf{W}_{\mathrm{E}}-\Gamma_{\text {tol }} \mathbf{V}\right) \\
\stackrel{(c)}{=} & \operatorname{Tr}(\tilde{\mathbf{L}}) \lambda_{\max }\left(\mathbf{W}-\Gamma_{\text {tol }} \mathbf{W}_{\mathrm{E}}-\Gamma_{\text {tol }} \mathbf{V}\right),
\end{array}
$$

where $(a)$ is due to the right hand side inequality in (30) while (b) and $(c)$ are due to the fact that $\tilde{\mathbf{L}}$ is a rank-one positive semi-definite matrix. Thus, by combining 27, 28), and (31), we obtain the following inequality:

$$
\begin{aligned}
& \operatorname{Pr}\left(\operatorname{Tr}\left(\tilde{\mathbf{L}}\left(\mathbf{W}-\Gamma_{\text {tol }} \mathbf{W}_{\mathrm{E}}-\Gamma_{\text {tol }} \mathbf{V}\right)\right) \leq \Gamma_{\text {tol }} \tilde{\sigma}^{2}\right) \\
& \geq \operatorname{Pr}\left(\lambda_{\max }\left(\mathbf{W}-\Gamma_{\text {tol }} \mathbf{W}_{\mathrm{E}}-\Gamma_{\text {tol }} \mathbf{V}\right) \operatorname{Tr}(\tilde{\mathbf{L}}) \leq \Gamma_{\text {tol }} \tilde{\sigma}^{2}\right) . \\
& \text { As a result, by setting } \operatorname{Pr}\left(\lambda _ { \operatorname { m a x } } \left(\mathbf{W}-\Gamma_{\text {tol }} \mathbf{W}_{\mathrm{E}}-\right.\right.
\end{aligned}
$$


$\left.\left.\Gamma_{\text {tol }} \mathbf{V}\right) \operatorname{Tr}(\tilde{\mathbf{L}}) \leq \Gamma_{\text {tol }} \tilde{\sigma}^{2}\right) \geq \kappa^{1 / J}$, we have

$$
\begin{aligned}
& \operatorname{Pr}\left(\lambda_{\max }(\mathbf{Q}) \operatorname{Tr}(\tilde{\mathbf{L}}) \leq \Gamma_{\mathrm{tol}} \tilde{\sigma}^{2}\right) \geq \kappa^{1 / J} \\
\stackrel{(d)}{\Longleftrightarrow} & \operatorname{Pr}\left(\frac{\lambda_{\max }(\mathbf{Q})}{\Gamma_{\mathrm{tol}} \tilde{\sigma}^{2}} \leq \frac{1}{\operatorname{Tr}(\tilde{\mathbf{L}})}\right) \geq \kappa^{1 / J} \\
\stackrel{(e)}{\Longleftrightarrow} & \operatorname{Pr}\left(\frac{\lambda_{\max }(\mathbf{Q})}{\Gamma_{\mathrm{tol}} \tilde{\sigma}^{2}} \geq \frac{1}{\operatorname{Tr}(\tilde{\mathbf{L}})}\right) \leq 1-\kappa^{1 / J} \\
\Longleftrightarrow & \Phi_{N_{\mathrm{T}}}^{-1}\left(1-\kappa^{1 / J}\right) \Gamma_{\mathrm{tol}} \tilde{\sigma}^{2} \geq \lambda_{\max }(\mathbf{Q}) \\
\Longleftrightarrow & \mathbf{I}_{\mathrm{N}_{\mathrm{T}}}\left(\Phi_{N_{\mathrm{T}}}^{-1}\left(1-\kappa^{1 / J}\right) \Gamma_{\mathrm{tol}} \tilde{\sigma}^{2}\right) \succeq \mathbf{Q} \\
\Longrightarrow & \operatorname{Pr}\left(\Gamma_{\mathrm{tol}} \tilde{\sigma}^{2} \geq \operatorname{Tr}(\tilde{\mathbf{L}} \mathbf{Q})\right) \geq \kappa^{1 / J} \\
\Longleftrightarrow & \operatorname{Pr}\left(\max _{j \in\{1, \ldots, J\}}\left\{\Gamma_{\mathrm{PE}, j}\right\} \leq \Gamma_{\mathrm{tol}}\right) \geq \kappa,
\end{aligned}
$$

where $\mathbf{Q}=\mathbf{W}-\Gamma_{\text {tol }} \mathbf{W}_{\mathrm{E}}-\Gamma_{\mathrm{tol}} \mathbf{V}$. $(d)$ and $(e)$ are due to the positive definiteness of matrix $\tilde{\mathbf{L}}$ and a basic property of probability, respectively. $\Phi_{N_{\mathrm{T}}}^{-1}(\cdot)$ in $33 \mathrm{~d}$ ) denotes the inverse cumulative distribution function (c.d.f.) of an inverse central chi-square random variable with $2 N_{\mathrm{T}}$ degrees of freedom. We note that the deviations in (33) do not require matrix $\mathbf{W}-$ $\Gamma_{\text {tol }} \mathbf{W}_{\mathrm{E}}-\Gamma_{\text {tol }} \mathbf{V}$ to be a positive semidefinite matrix. Besides, the non-smoothness of $\lambda_{\max }(\cdot)$ is overcome by replacing $33 \mathrm{~d}$ with its equivalent expression in (33e). Furthermore, random variable $\tilde{\mathbf{L}}$ is decoupled from the optimization variables, cf. (31). Thus, the implication in 33d is applicable to any continuous channel distribution by replacing $\Phi_{N_{\mathrm{T}}}^{-1}(\cdot)$ with an inverse c.d.f. with respect to the corresponding distribution. In practice, the inverse function of the inverse central chi-square c.d.f. can be evaluated directly or be stored in a lookup table in a practical implementation.

\section{B. Proof of Theorem 1}

The proof of Theorem 1 utilizes the results from [17, Proposition 4.1]. In the first part, we show the structure of the optimal solution $\mathbf{W}^{*}$. Then, in the second part, we propose a method for constructing a solution $\left\{\widetilde{\mathbf{W}}^{*}, \widetilde{\mathbf{V}}^{*}, \widetilde{\mathbf{W}}_{\mathrm{E}}^{*}, \widetilde{\rho}^{*}, \widetilde{\boldsymbol{\delta}}^{*}, \widetilde{\boldsymbol{\nu}}^{*}\right\}$ that achieves the same objective value as $\left\{\mathbf{W}^{*}, \mathbf{V}^{*}, \mathbf{W}_{\mathrm{E}}^{*}, \rho^{*}, \boldsymbol{\delta}^{*}, \boldsymbol{\nu}^{*}\right\}$ and admits a rank-one $\widetilde{\mathbf{W}}^{*}$. It can be shown that the relaxed version of problem 25 is jointly convex with respect to the optimization variables and satisfies Slater's constraint qualification. As a result, the Karush-Kuhn-Tucker (KKT) conditions are necessary and sufficient conditions [28] for the solution of the relaxed problem. The Lagrangian function of (25) is given by

$$
\begin{aligned}
\mathcal{L} & =\operatorname{Tr}(\mathbf{W})-\operatorname{Tr}(\mathbf{W} \mathbf{Y})-\sum_{k=1}^{K-1} \operatorname{Tr}\left(\mathbf{S}_{\mathrm{C}_{2_{k}}}\left(\mathbf{W}, \mathbf{V}, \delta_{k}\right) \mathbf{D}_{\mathrm{C}_{2_{k}}}\right) \\
& -\sum_{k=1}^{K-1} \operatorname{Tr}\left(\mathbf{S}_{\mathrm{C}_{5_{k}}}\left(\mathbf{W}, \mathbf{V}, \mathbf{W}_{\mathrm{E}}, \nu_{k}\right) \mathbf{D}_{\mathrm{C}_{5_{k}}}\right) \\
& +\mu\left(\frac{P_{\min }}{\eta(1-\rho)}-\operatorname{Tr}(\mathbf{H W})\right)+\beta\left(\frac{\Gamma_{\mathrm{req}} \sigma_{\mathrm{s}}^{2}}{\rho}-\operatorname{Tr}(\mathbf{H W})\right) \\
& +\sum_{n=1}^{N_{\mathrm{T}}} \theta_{n} \operatorname{Tr}\left(\mathbf{\Psi}_{n} \mathbf{W}\right)+\operatorname{Tr}\left(\mathbf{W} \mathbf{D}_{\overline{\mathrm{C}_{3}}}\right)+\Delta,
\end{aligned}
$$

where $\Delta$ denotes the collection of terms that only involve variables that are not relevant for the proof. In (34), $\beta \geq 0$ is the Lagrange multiplier for the minimum required SINR of the desired receiver in $\mathrm{C} 1 . \mathbf{D}_{\mathrm{C}_{2_{k}}} \succeq \mathbf{0}, \forall k \in\{1, \ldots, K-$ $1\}, \mathbf{D}_{\overline{\mathrm{C}_{3}}} \succeq \mathbf{0}$, and $\mathbf{D}_{\mathrm{C}_{5_{k}}} \succeq \mathbf{0}, \forall k \in\{1, \ldots, K-1\}$, are the Lagrange multiplier matrices corresponding to constraints $\mathrm{C} 2, \overline{\mathrm{C} 3}$, and $\mathrm{C} 5$, respectively. Lagrange multiplier $\mu \geq 0$ corresponds to the minimum required power transfer to the desired receiver in $\mathrm{C} 4 . \boldsymbol{\theta}$ is the Lagrange multiplier vector corresponding to constraint $\mathrm{C} 6$ on the per-antenna maximum transmit power with elements $\theta_{n} \geq 0, \forall n \in\left\{1, \ldots, N_{\mathrm{T}}\right\}$. Matrix $\mathbf{Y} \succeq \mathbf{0}$ is the Lagrange multiplier matrix for the positive semi-definite constraint on matrix $\mathbf{W}$ in $\mathrm{C} 8$. Considering (34), the KKT conditions for the optimal $\mathbf{W}^{*}$ are given by:

$$
\begin{aligned}
\mathbf{Y}^{*}, \mathbf{D}_{\mathrm{C}_{2_{k}}}^{*}, \mathbf{D}_{\mathrm{C}_{3}}^{*}, \mathbf{D}_{\mathrm{C}_{5_{k}}}^{*} & \succeq \mathbf{0}, \quad \mu^{*}, \beta^{*}, \theta_{n}^{*} \geq 0, \\
\mathbf{Y}^{*} \mathbf{W}^{*} & =\mathbf{0}, \\
\nabla_{\mathbf{W}} \mathcal{L} & =\mathbf{0}, \quad \sqrt{\beta^{*} \sigma_{\mathrm{s}}^{2} \Gamma_{\text {req }}} \\
\frac{\partial \mathcal{L}}{\partial \rho^{*}}=0 \Rightarrow \rho^{*} & =\frac{\sqrt{\beta^{*} \sigma_{\mathrm{s}}^{2} \Gamma_{\text {req }}}+\sqrt{\frac{\mu^{*} P_{\min }}{\eta}}}{}
\end{aligned}
$$

where $\mathbf{Y}^{*}, \mathbf{D}_{\mathrm{C}_{2_{k}}}^{*}, \mathbf{D}_{\overline{\mathrm{C}}_{3}}^{*}, \mathbf{D}_{\mathrm{C}_{5_{k}}}^{*}, \mu^{*}, \beta^{*}$ and $\theta_{n}^{*}$, are the optimal Lagrange multipliers for the dual problem of 25). It can be observed from (38) that constraint C7: $0 \leq \rho \leq 1$ is automatically satisfied. Besides, $\mu^{*}, \beta^{*}>0$ must holds for $\Gamma_{\text {req }}>0$ and $P^{\min }>0$. On the other hand, 36 is the complementary slackness condition and is satisfied when the columns of $\mathbf{W}^{*}$ lie in the null space of $\mathbf{Y}^{*}$. Thus, the KKT condition in 37) can be expressed as

$$
\begin{aligned}
& \mathbf{I}_{N_{\mathrm{T}}}+\mathbf{D}_{\overline{\mathrm{C}}_{3}}^{*}+\sum_{n=1}^{N_{\mathrm{T}}} \theta_{n}^{*} \mathbf{\Psi}_{n}+\sum_{k=1}^{K-1} \mathbf{U}_{\mathbf{g}_{k}}\left(\frac{\mathbf{D}_{\mathrm{C}_{2_{k}}}^{*}}{\Gamma_{\mathrm{tol}_{k}}}-\mathbf{D}_{\mathrm{C}_{5_{k}}}^{*}\right) \mathbf{U}_{\mathbf{g}_{k}}^{H} \\
= & \mathbf{Y}^{*}+\left(\mu^{*}+\beta^{*}\right) \mathbf{H} .
\end{aligned}
$$

For notational simplicity, we define

$$
\begin{aligned}
\mathbf{B}^{*} & =\mathbf{I}_{N_{\mathrm{T}}}+\mathbf{D}_{\mathrm{C}_{3}}^{*}+\sum_{n=1}^{N_{\mathrm{T}}} \theta_{n}^{*} \mathbf{\Psi}_{n} \\
& +\sum_{k=1}^{K-1} \mathbf{U}_{\mathbf{g}_{k}}\left(\frac{\mathbf{D}_{\mathrm{C}_{2_{k}}}^{*}}{\Gamma_{\mathrm{tol}_{k}}}-\mathbf{D}_{\mathrm{C}_{5_{k}}}^{*}\right) \mathbf{U}_{\mathbf{g}_{k}}^{H} .
\end{aligned}
$$

From the complementary slackness condition in (36), the columns of $\mathbf{W}^{*}$ have to lie in the null space of $\mathbf{Y}^{*}$ for $\mathbf{W}^{*} \neq \mathbf{0}$. Thus, we study the rank and null space of $\mathbf{Y}^{*}$ for obtaining the structure of the optimal solution $\mathbf{W}^{*}$. From (39) and (40), we obtain for the Lagrange multiplier matrix

$$
\mathbf{Y}^{*}=\mathbf{B}^{*}-\left(\mu^{*}+\beta^{*}\right) \mathbf{H},
$$

where $\left(\mu^{*}+\beta^{*}\right) \mathbf{H}$ is a rank-one matrix since $\mu^{*}, \beta^{*}>0$. Without loss of generality, we define $r=\operatorname{Rank}\left(\mathbf{B}^{*}\right)$ and the orthonormal basis of the null space of $\mathbf{B}^{*}$ as $\mathbf{\Upsilon} \in$ $\mathbb{C}^{N_{\mathrm{T}} \times\left(N_{\mathrm{T}}-r\right)}$ such that $\mathbf{B}^{*} \boldsymbol{\Upsilon}=\mathbf{0}$ and $\operatorname{Rank}(\mathbf{\Upsilon})=N_{\mathrm{T}}-r$. Let $\varrho_{t} \in \mathbb{C}^{N_{\mathrm{T}} \times 1}, 1 \leq t \leq N_{\mathrm{T}}-r$, denote the $t$-th column of $\Upsilon$. By exploiting [17, Proposition 4.1], it can be shown that $\mathbf{H} \Upsilon=\mathbf{0}$ and we can express the optimal solution of $\mathbf{W}^{*}$ as

$$
\mathbf{W}^{*}=\sum_{t=1}^{N_{\mathrm{T}}-r} \gamma_{t} \boldsymbol{\varrho}_{t} \boldsymbol{\varrho}_{t}^{H}+f \mathbf{u u}^{H},
$$




$$
\begin{aligned}
& \text { Objective value: } \operatorname{Tr}\left(\widetilde{\mathbf{W}}^{*}\right)+\operatorname{Tr}\left(\widetilde{\mathbf{V}}^{*}\right)+\operatorname{Tr}\left(\widetilde{\mathbf{W}}_{\mathrm{E}}^{*}\right)=\operatorname{Tr}\left(\mathbf{W}^{*}\right)+\operatorname{Tr}\left(\mathbf{V}^{*}\right)+\operatorname{Tr}\left(\mathbf{W}_{\mathrm{E}}^{*}\right) \\
& \mathrm{C} 1: \frac{\operatorname{Tr}\left(\widetilde{\mathbf{W}}^{*} \mathbf{H}\right)}{\sigma_{\text {ant }}^{2}+\operatorname{Tr}\left(\mathbf{H} \widetilde{\mathbf{V}}^{*}\right)+\frac{\sigma_{s}^{2}}{\rho}}=\frac{\operatorname{Tr}\left(\left(\mathbf{W}^{*}-\sum_{t=1}^{N_{\mathrm{T}}-r} \gamma_{t} \varrho_{t} \varrho_{t}^{H}\right) \mathbf{H}\right)}{\sigma_{\text {ant }}^{2}+\operatorname{Tr}\left(\mathbf{H}\left(\mathbf{V}^{*}+\sum_{t=1}^{N_{\mathrm{T}}-r} \gamma_{t} \varrho_{t} \boldsymbol{\varrho}_{t}^{H}\right)\right)+\frac{\sigma_{\mathrm{s}}^{2}}{\rho}} \\
& =\frac{\operatorname{Tr}\left(\mathbf{W}^{*} \mathbf{H}\right)}{\sigma_{\text {ant }}^{2}+\operatorname{Tr}\left(\mathbf{H V}^{*}\right)+\frac{\sigma_{\mathrm{s}}^{2}}{\rho}} \geq \Gamma_{\text {req }}, \\
& \text { C2: } \mathbf{S}_{\mathrm{C}_{2_{k}}}\left(\widetilde{\mathbf{W}}^{*}, \widetilde{\mathbf{V}}^{*}, \widetilde{\delta}_{k}\right) \succeq \mathbf{S}_{\mathrm{C}_{2_{k}}}\left(\mathbf{W}^{*}, \mathbf{V}^{*}, \delta_{k}\right)+\mathbf{U}_{\mathbf{g}_{k}}^{H}\left[\sum_{t=1}^{N_{\mathrm{T}}-r} \gamma_{t} \varrho_{t} \boldsymbol{\varrho}_{t}^{H}\right] \mathbf{U}_{\mathbf{g}_{k}}\left(1+\frac{1}{\Gamma_{\mathrm{tol}_{k}}}\right) \succeq \mathbf{0}, \forall k, \\
& \overline{\mathrm{C} 3}: \lambda_{\max }\left(\widetilde{\mathbf{W}}^{*}-\Gamma_{\mathrm{tol}} \widetilde{\mathbf{W}}_{\mathrm{E}}^{*}-\Gamma_{\mathrm{tol}} \widetilde{\mathbf{V}}^{*}\right) \leq \lambda_{\max }\left(\mathbf{W}^{*}-\Gamma_{\mathrm{tol}} \mathbf{W}_{\mathrm{E}}^{*}-\Gamma_{\mathrm{tol}} \mathbf{V}^{*}\right) \leq \Gamma_{\mathrm{tol}} \tilde{\sigma}^{2} \Phi_{N_{\mathrm{T}}}^{-1}\left(1-\kappa^{1 / J}\right), \\
& \text { C4: } \operatorname{Tr}\left(\mathbf{H} \widetilde{\mathbf{W}}^{*}\right)+\operatorname{Tr}\left(\mathbf{H} \widetilde{\mathbf{W}}_{\mathrm{E}}^{*}\right)+\operatorname{Tr}\left(\mathbf{H} \widetilde{\mathbf{V}}^{*}\right)+\sigma_{\text {ant }}^{2} \\
& =\operatorname{Tr}\left(\mathbf{H} \mathbf{W}^{*}\right)+\operatorname{Tr}\left(\mathbf{H} \mathbf{W}_{\mathrm{E}}^{*}\right)+\operatorname{Tr}\left(\mathbf{H} \mathbf{V}^{*}\right)+\sigma_{\text {ant }}^{2} \geq \frac{P_{\min }}{\left(1-\rho^{*}\right) \eta}=\frac{P_{\min }}{\left(1-\tilde{\rho}^{*}\right) \eta}, \\
& \text { C5: } \mathbf{S}_{\mathrm{C}_{5_{k}}}\left(\widetilde{\mathbf{W}}^{*}, \widetilde{\mathbf{V}}^{*}, \widetilde{\mathbf{W}}_{\mathrm{E}}^{*}, \widetilde{\nu}_{k}^{*}\right)=\mathbf{S}_{\mathrm{C}_{5_{k}}}\left(\mathbf{W}^{*}, \mathbf{V}^{*}, \mathbf{W}_{\mathrm{E}}^{*}, \nu_{k}^{*}\right) \succeq \mathbf{0}, \forall k, \\
& \text { C6: } \operatorname{Tr}\left(\boldsymbol{\Psi}_{n}\left(\mathbf{W}^{*}+\mathbf{V}^{*}+\mathbf{W}_{\mathrm{E}}^{*}\right)\right)=\operatorname{Tr}\left(\boldsymbol{\Psi}_{n}\left(\widetilde{\mathbf{W}}^{*}+\widetilde{\mathbf{V}}^{*}+\widetilde{\mathbf{W}}_{\mathrm{E}}^{*}\right)\right) \leq P_{\max _{n}}, \forall n, \\
& \text { C7: } 0 \leq \widetilde{\rho}^{*} \leq 1, \quad \mathrm{C} 8: \widetilde{\mathbf{W}}^{*} \succeq \mathbf{0}, \widetilde{\mathbf{V}}^{*} \succeq \mathbf{0}, \widetilde{\mathbf{W}}_{\mathrm{E}}^{*} \succeq \mathbf{0}, \mathbf{C} 10: \widetilde{\delta}_{k}^{*}, \widetilde{\nu}_{k}^{*} \geq 0, \forall k \text {. }
\end{aligned}
$$

where $\gamma_{t} \geq 0, \forall t, f>0, f \in \mathbb{R}$, are positive scaling constants and $\mathbf{u} \in \mathbb{C}^{N_{\mathrm{T}} \times 1},\|\mathbf{u}\|=1$, satisfies $\mathbf{u}^{H} \boldsymbol{\Upsilon}=\mathbf{0}$.

In the second part of the proof, for $\operatorname{Rank}\left(\mathbf{W}^{*}\right)>1$, we construct another solution of the relaxed version of problem (25), $\left\{\widetilde{\mathbf{W}}^{*}, \widetilde{\mathbf{V}}^{*}, \widetilde{\mathbf{W}}_{\mathrm{E}}^{*}, \widetilde{\rho}^{*}, \widetilde{\boldsymbol{\delta}}^{*}, \widetilde{\boldsymbol{\nu}}^{*}\right\}$, based on (42). Let

$$
\begin{aligned}
\widetilde{\mathbf{W}}^{*} & =f \mathbf{u} \mathbf{u}^{H}=\mathbf{W}^{*}-\sum_{t=1}^{N_{\mathrm{T}}-r} \gamma_{t} \boldsymbol{\varrho}_{t} \boldsymbol{\varrho}_{t}^{H}, \\
\widetilde{\mathbf{V}}^{*} & =\mathbf{V}^{*}+\sum_{t=1}^{N_{\mathrm{T}}-r} \gamma_{t} \boldsymbol{\varrho}_{t} \boldsymbol{\varrho}_{t}^{H}, \\
\widetilde{\mathbf{W}}_{\mathrm{E}}^{*} & =\mathbf{W}_{\mathrm{E}}^{*}, \quad \widetilde{\rho}^{*}=\rho^{*}, \quad \widetilde{\boldsymbol{\delta}}^{*}=\boldsymbol{\delta}^{*}, \text { and } \widetilde{\boldsymbol{\nu}}^{*}=\boldsymbol{\nu}^{*} .
\end{aligned}
$$

Then, we substitute $\left\{\widetilde{\mathbf{W}}^{*}, \widetilde{\mathbf{V}}^{*}, \widetilde{\mathbf{W}}_{\mathrm{E}}^{*}, \widetilde{\rho}^{*}, \widetilde{\boldsymbol{\delta}}^{*}, \widetilde{\boldsymbol{\nu}}^{*}\right\}$ into the objective function and constraints in (25) which yields the equations (45)-(52) on the top of this page, where (48) is due to 33d and 33e. It can be seen from (45) that the solution set $\left\{\widetilde{\mathbf{W}}^{*}, \widetilde{\mathbf{V}}^{*}, \widetilde{\mathbf{W}}_{\mathrm{E}}^{*}, \widetilde{\rho}^{*}, \widetilde{\boldsymbol{\delta}}^{*}, \widetilde{\boldsymbol{\nu}}^{*}\right\}$ achieves the same optimal value as the optimal solution $\left\{\mathbf{W}^{*}, \mathbf{V}^{*}, \mathbf{W}_{\mathrm{E}}^{*}, \rho^{*}, \boldsymbol{\delta}^{*}, \boldsymbol{\nu}^{*}\right\}$ and satisfies all the constraints, cf. (46)-(52). Thus, $\left\{\widetilde{\mathbf{W}}^{*}, \widetilde{\mathbf{V}}^{*}, \widetilde{\mathbf{W}}_{\mathrm{E}}^{*}, \widetilde{\rho}^{*}, \widetilde{\boldsymbol{\delta}}^{*}, \widetilde{\boldsymbol{\nu}}^{*}\right\}$ is also an optimal solution but with $\operatorname{Rank}\left(\widetilde{\mathbf{W}}^{*}\right)=1$, cf. (43).

Remark 6: We note that $\left\{\widetilde{\mathbf{W}}^{*}, \widetilde{\mathbf{V}}^{*}, \widetilde{\mathbf{W}}_{\mathrm{E}}^{*}, \widetilde{\rho}^{*}, \widetilde{\boldsymbol{\delta}}^{*}, \widetilde{\boldsymbol{\nu}}^{*}\right\}$ can be obtained by substituting (43) and (44) into the relaxed version of (25). Then, we solve the resulting convex optimization problem for $f$ and $\gamma_{t}$. On the other hand, the constructed solution set with $\operatorname{Rank}\left(\widetilde{\mathbf{W}}^{*}\right)=1$ is not unique. In [43), we construct $\widetilde{\mathbf{V}}^{*}$ by modifying the artificial noise structure of the original solution, $\mathbf{V}^{*}$, such that the constructed beamforming matrix $\widetilde{\mathbf{W}}^{*}$ is rank-one. In fact, for achieving a rank-one $\widetilde{\mathbf{W}}^{*}$, a general structure for constructing the artificial noise and the energy signal can be expressed as $\widetilde{\mathbf{V}}^{*}=\mathbf{V}^{*}+$ $\sum_{t=1}^{N_{\mathrm{T}}-r} \pi_{t} \gamma_{t} \varrho_{t} \varrho_{t}^{H}$ and $\widetilde{\mathbf{W}}_{\mathrm{E}}^{*}=\mathbf{W}_{\mathrm{E}}^{*}+\sum_{t=1}^{N_{\mathrm{T}}-r}\left(1-\pi_{t}\right) \gamma_{t} \varrho_{t} \varrho_{t}^{H}$, respectively, for any $0 \leq \pi_{t} \leq 1, \forall t \in\left\{1, \ldots, N_{\mathrm{T}}-r\right\}$. Yet, the non-uniqueness of the constructed solution does not play an important role in this paper since all solutions achieve the same optimal objective value while satisfying all the constraints.

\section{Proof of Proposition 1}

We prove that $\operatorname{Rank}\left(\mathbf{W}^{*}\right)=1$ when $\frac{\mathbf{D}_{\mathrm{C}_{2}}^{*}}{\Gamma_{\text {tol }}}-\mathbf{D}_{\mathrm{C}_{5_{k}}}^{*} \succeq \mathbf{0}, \forall k$ holds. First, we post-multiply both sides of (41) by $\mathbf{W}^{*}$ which yields

$$
\begin{aligned}
\mathbf{B}^{*} \mathbf{W}^{*} & =\left(\mathbf{Y}^{*}+\left(\mu^{*}+\beta^{*}\right) \mathbf{H}\right) \mathbf{W}^{*} \\
& \stackrel{(a)}{=}\left(\mu^{*}+\beta^{*}\right) \mathbf{H} \mathbf{W}^{*},
\end{aligned}
$$

where $(a)$ is due to the complementary slackness condition in (36). Since $\frac{\mathbf{D}_{\mathrm{C}_{2}}^{*}}{\Gamma_{\mathrm{tol}_{k}}}-\mathbf{D}_{\mathrm{C}_{5_{k}}}^{*} \succeq \mathbf{0}, \forall k$ holds by assumption, $\mathbf{B}^{*}$ is a positive definite matrix. Then, by exploiting (53) and a basic rank inequality, we have

$$
\begin{aligned}
\operatorname{Rank}\left(\mathbf{W}^{*}\right) & =\operatorname{Rank}\left(\mathbf{B}^{*} \mathbf{W}^{*}\right)=\operatorname{Rank}\left(\left(\mu^{*}+\beta^{*}\right) \mathbf{H} \mathbf{W}^{*}\right) \\
& =\min \left\{\operatorname{Rank}\left(\mathbf{W}^{*}\right), \operatorname{Rank}\left(\left(\mu^{*}+\beta^{*}\right) \mathbf{H}\right)\right\} .(54)
\end{aligned}
$$

We note that $\operatorname{Rank}(\mathbf{H})=1$ and $\operatorname{Rank}\left(\left(\mu^{*}+\beta^{*}\right) \mathbf{H}\right)$ is equal to one since $\mu^{*}+\beta^{*}>0$. On the other hand, $\mathbf{W}^{*} \neq \mathbf{0}$ is required to satisfy the minimum SINR requirement of the desired receiver in $\mathrm{C} 1$ when $\Gamma_{\text {req }}>0$. Therefore, $\operatorname{Rank}\left(\left(\mu^{*}+\right.\right.$ $\left.\left.\beta^{*}\right) \mathbf{H}\right)=1$ and $\operatorname{Rank}\left(\mathbf{W}^{*}\right)=1$ when $\frac{\mathbf{D}_{\mathrm{C}_{k}}^{*}}{\Gamma_{\mathrm{tol}_{k}}}-\mathbf{D}_{\mathrm{C}_{5_{k}}}^{*} \succeq \mathbf{0}, \forall k$ holds.

\section{REFERENCES}

[1] D. W. K. Ng and R. Schober, "Resource Allocation for Secure Communication in Systems with Wireless Information and Power Transfer,' in Proc. IEEE Global Telecommun. Conf., Dec. 2013.

[2] D. W. K. Ng, L. Xiang, and R. Schober, "Multi-Objective Beamforming for Secure Communication in Systems with Wireless Information and Power Transfer," in Proc. IEEE Intern. Personal, Indoor and Mobile Radio Commun. Sympos., Sep. 2013.

[3] L. Varshney, "Transporting Information and Energy Simultaneously," in Proc. IEEE Intern. Sympos. on Inf. Theory, Jul. 2008, pp. $1612-1616$. 
[4] P. Grover and A. Sahai, "Shannon Meets Tesla: Wireless Information and Power Transfer," in Proc. IEEE Intern. Sympos. on Inf. Theory, Jun. 2010, pp. $2363-2367$.

[5] X. Zhou, R. Zhang, and C. K. Ho, "Wireless Information and Power Transfer: Architecture Design and Rate-Energy Tradeoff," in Proc. IEEE Global Telecommun. Conf., Dec. 2012.

[6] R. Zhang and C. K. Ho, "MIMO Broadcasting for Simultaneous Wireless Information and Power Transfer," IEEE Trans. Wireless Commun., vol. 12, pp. 1989-2001, May 2013.

[7] D. W. K. Ng, E. S. Lo, and R. Schober, "Wireless Information and Power Transfer: Energy Efficiency Optimization in OFDMA Systems," IEEE Trans. Wireless Commun., vol. 12, pp. 6352 - 6370, Dec. 2013.

[8] J. Xu, L. Liu, and R. Zhang, "Multiuser MISO Beamforming for Simultaneous Wireless Information and Power Transfer," in Proc. IEEE Intern. Conf. on Acoust., Speech and Signal Process., May 2013.

[9] T. Chen, Y. Yang, H. Zhang, H. Kim, and K. Horneman, "Network Energy Saving Technologies for Green Wireless Access Networks," IEEE Wireless Commun., vol. 18, pp. 30-38, Oct. 2011.

[10] R. Liu and W. Trappe, Securing Wireless Communications at the Physical Layer. New York: Springer, 2009.

[11] M. Bloch and J. Barros, Physical-Layer Security: From Information Theory to Security Engineering, 1st ed. Cambridge University Press, 2011.

[12] A. D. Wyner, "The Wire-Tap Channel," Tech. Rep., Oct. 1975.

[13] S. Goel and R. Negi, "Guaranteeing Secrecy using Artificial Noise," IEEE Trans. Wireless Commun., vol. 7, pp. 2180 - 2189, Jun. 2008.

[14] D. W. K. Ng, E. S. Lo, and R. Schober, "Secure Resource Allocation and Scheduling for OFDMA Decode-and-Forward Relay Networks," IEEE Trans. Wireless Commun., vol. 10, pp. 3528-3540, Oct. 2011.

[15] W.-C. Liao, T.-H. Chang, W.-K. Ma, and C.-Y. Chi, "QoS-Based Transmit Beamforming in the Presence of Eavesdroppers: An Optimized Artificial-Noise-Aided Approach," IEEE Trans. Signal Process., vol. 59, pp. 1202-1216, Mar. 2011.

[16] Q. Li, W.-K. Ma, and A.-C. So, "Safe Convex Approximation to OutageBased MISO Secrecy Rate Optimization under Imperfect CSI and with Artificial Noise," in Proc. ASILOMAR Conf. on Comput. Commun. Signals, Syst. and Computers, Nov. 2011, pp. 207-211.

[17] L. Liu, R. Zhang, and K.-C. Chua, "Secrecy Wireless Information and Power Transfer with MISO Beamforming," IEEE Trans. Signal Process., vol. 62, pp. $1850-1863$, Apr. 2014.

[18] — , "Secrecy Wireless Information and Power Transfer with MISO Beamforming," in Proc. IEEE Global Telecommun. Conf., Dec. 2013.

[19] Z. Xiang and M. Tao, "Robust Beamforming for Wireless Information and Power Transmission," IEEE Wireless Commun. Lett., vol. 1, pp. 372 -375 , Aug. 2012.

[20] H. Jabbar, Y. Song, and T. Jeong, "RF Energy Harvesting System and Circuits for Charging of Mobile Devices," IEEE Trans. on Consum. Electron., vol. 56, pp. 247-253, Feb. 2010.

[21] G. Zheng, K. K. Wong, and T. S. Ng, "Robust Linear MIMO in the Downlink: A Worst-Case Optimization with Ellipsoidal Uncertainty Regions," EURASIP J. Adv. Signal Process., vol. 2008, 2008, Article ID 609028.

[22] C. Shen, T.-H. Chang, K.-Y. Wang, Z. Qiu, and C.-Y. Chi, "Distributed Robust Multicell Coordinated Beamforming With Imperfect CSI: An ADMM Approach," IEEE Trans. Signal Process., vol. 60, pp. 2988 3003, Jun. 2012

[23] Q. Wang, H. Su, K. Ren, and K. Kim, "Fast and Scalable Secret Key Generation Exploiting Channel Phase Randomness in Wireless Networks," in Proc. IEEE Intern. Conf. on Comput. Commun., Apr. 2011, pp. 1422-1430.

[24] M. Shenouda and T. Davidson, "Outage-Based Designs for Multi-user Transceivers," in Proc. IEEE Intern. Conf. on Acoust., Speech and Signal Process., Apr. 2009, pp. 2389-2392.

[25] K.-Y. Wang, A. M.-C. So, T.-H. Chang, W.-K. Ma, and C.-Y. Chi, "Outage Constrained Robust Transmit Optimization for Multiuser MISO Downlinks: Tractable Approximations by Conic Optimization," 2011. [Online]. Available: http://arxiv.org/abs/1108.0982

[26] M. Bengtsson and B. Ottersten, "Optimal Downlink Beamforming using Semidefinite Optimization," in Proc. Annual Allerton Conf. on Commun., Control and Computing, Sep. 1999, pp. 987-996.

[27] Z.-Q. Luo, W.-K. Ma, A.-C. So, Y. Ye, and S. Zhang, "Semidefinite Relaxation of Quadratic Optimization Problems," IEEE Signal Process. Mag., vol. 27, pp. 20-34, May 2010.

[28] S. Boyd and L. Vandenberghe, Convex Optimization. Cambridge University Press, 2004.

[29] D. Tse and P. Viswanath, Fundamentals of Wireless Communication, 1st ed. Cambridge University Pres, 2005.
[30] Q. Li and W. K. Ma, "Spatically Selective Artificial-Noise Aided Transmit Optimization for MISO Multi-Eves Secrecy Rate Maximization," IEEE Trans. Signal Process., vol. 61, pp. 2704-2717, May 2013.

[31] N. Romero-Zurita, M. Ghogho, and D. McLernon, "Outage Probability Based Power Distribution Between Data and Artificial Noise for Physical Layer Security," IEEE Signal Process. Lett., vol. 19, pp. 71-74, Feb. 2012.

[32] J. F. Sturm, "Using SeDuMi 1.02, A MATLAB Toolbox for Optimization over Symmetric Cones," Optimiz. Methods and Softw., vol. 11-12, pp. 625-653, Sep. 1999.

[33] K. C. Toh, M. J. Todd, and R. H. Tütüncü, "SDPT3 - A Matlab Software Package for Semidefinite Programming, Version 1.3," Optimization Methods and Softw., vol. 11, pp. 545-581, Jan. 1999.

[34] IEEE P802.11 Wireless LANs, "TGn Channel Models", IEEE 802.1103/940r4, Tech. Rep., May 2004.

[35] H.-S. Chen and W. Gao, "MAC and PHY Proposal for 802.11af," Tech. Rep., Feb., [Online] https://mentor.ieee.org/802.11/dcn/10/11-10-0258-00-00af-mac-and-phy-proposal-for-

[36] J. B. Lasserre, "A Trace Inequality for Matrix Product," IEEE Trans. Autom. Control, vol. 40, pp. 1500-1501, Aug. 1995. 